University of Nebraska - Lincoln

DigitalCommons@University of Nebraska - Lincoln

Faculty Publications from the Department of Electrical \& Computer Engineering, Department Electrical and Computer Engineering

10-15-2003

\title{
VECTOR DISSIPATIVITY THEORY FOR DISCRETE-TIME LARGE- SCALE NONLINEAR DYNAMICAL SYSTEMS
}

Wassim M. Haddad

Georgia Institute of Technology, wm.haddad@aerospace.gatech.edu

Qing Hui

Georgia Institute of Technology, qing.hui@unl.edu

VijaySekhar Chellaboina

University of Missouri-Columbia, chellaboinav@missouri.edu

Sergey Nersesov

Georgia Institute of Technology, sergeinersesov@ae.gatech.edu

Follow this and additional works at: https://digitalcommons.unl.edu/electricalengineeringfacpub

Part of the Computer Engineering Commons, and the Electrical and Computer Engineering Commons

Haddad, Wassim M.; Hui, Qing; Chellaboina, VijaySekhar; and Nersesov, Sergey, "VECTOR DISSIPATIVITY THEORY FOR DISCRETE-TIME LARGE-SCALE NONLINEAR DYNAMICAL SYSTEMS" (2003). Faculty Publications from the Department of Electrical and Computer Engineering. 522.

https://digitalcommons.unl.edu/electricalengineeringfacpub/522

This Article is brought to you for free and open access by the Electrical \& Computer Engineering, Department of at DigitalCommons@University of Nebraska - Lincoln. It has been accepted for inclusion in Faculty Publications from the Department of Electrical and Computer Engineering by an authorized administrator of DigitalCommons@University of Nebraska - Lincoln. 


\title{
VECTOR DISSIPATIVITY THEORY FOR DISCRETE-TIME LARGE-SCALE NONLINEAR DYNAMICAL SYSTEMS
}

\author{
WASSIM M. HADDAD, QING HUI, VIJAYSEKHAR CHELLABOINA, \\ AND SERGEY NERSESOV
}

Received 15 October 2003

In analyzing large-scale systems, it is often desirable to treat the overall system as a collection of interconnected subsystems. Solution properties of the large-scale system are then deduced from the solution properties of the individual subsystems and the nature of the system interconnections. In this paper, we develop an analysis framework for discrete-time large-scale dynamical systems based on vector dissipativity notions. Specifically, using vector storage functions and vector supply rates, dissipativity properties of the discrete-time composite large-scale system are shown to be determined from the dissipativity properties of the subsystems and their interconnections. In particular, extended Kalman-Yakubovich-Popov conditions, in terms of the subsystem dynamics and interconnection constraints, characterizing vector dissipativeness via vector system storage functions are derived. Finally, these results are used to develop feedback interconnection stability results for discrete-time large-scale nonlinear dynamical systems using vector Lyapunov functions.

\section{Introduction}

Modern complex dynamical systems are highly interconnected and mutually interdependent, both physically and through a multitude of information and communication network constraints. The sheer size (i.e., dimensionality) and complexity of these large-scale dynamical systems often necessitate a hierarchical decentralized architecture for analyzing and controlling these systems. Specifically, in the analysis and control-system design of complex large-scale dynamical systems, it is often desirable to treat the overall system as a collection of interconnected subsystems. The behavior of the aggregate or composite (i.e., large-scale) system can then be predicted from the behaviors of the individual subsystems and their interconnections. The need for decentralized analysis and control design of large-scale systems is a direct consequence of the physical size and complexity of the dynamical model. In particular, computational complexity may be too large for model analysis while severe constraints on communication links between system sensors, actuators, and processors may render centralized control architectures impractical. 
An approach to analyzing large-scale dynamical systems was introduced by the pioneering work of Šiljak [19] and involves the notion of connective stability. In particular, the large-scale dynamical system is decomposed into a collection of subsystems with local dynamics and uncertain interactions. Then, each subsystem is considered independently so that the stability of each subsystem is combined with the interconnection constraints to obtain a vector Lyapunov function for the composite large-scale dynamical system guaranteeing connective stability for the overall system. Vector Lyapunov functions were first introduced by Bellman [2] and Matrosov [17] and further developed by Lakshmikantham et al. [11], with $[7,14,15,16,18,19,20]$ exploiting their utility for analyzing largescale systems. The use of vector Lyapunov functions in large-scale system analysis offers a very flexible framework since each component of the vector Lyapunov function can satisfy less-rigid requirements as compared to a single scalar Lyapunov function. Moreover, in large-scale systems, several Lyapunov functions arise naturally from the stability properties of each subsystem. An alternative approach to vector Lyapunov functions for analyzing large-scale dynamical systems is an input-output approach wherein stability criteria are derived by assuming that each subsystem is either finite gain, passive, or conic $[1,12,13,21]$.

Since most physical processes evolve naturally in continuous time, it is not surprising that the bulk of large-scale dynamical system theory has been developed for continuoustime systems. Nevertheless, it is the overwhelming trend to implement controllers digitally. Hence, in this paper we extend the notions of dissipativity theory $[22,23]$ to develop vector dissipativity notions for large-scale nonlinear discrete-time dynamical systems; a notion not previously considered in the literature. In particular, we introduce a generalized definition of dissipativity for large-scale nonlinear discrete-time dynamical systems in terms of a vector inequality involving a vector supply rate, a vector storage function, and a nonnegative, semistable dissipation matrix. Generalized notions of vector available storage and vector required supply are also defined and shown to be elementby-element ordered, nonnegative, and finite. On the subsystem level, the proposed approach provides a discrete energy flow balance in terms of the stored subsystem energy, the supplied subsystem energy, the subsystem energy gained from all other subsystems independent of the subsystem coupling strengths, and the subsystem energy dissipated. Furthermore, for large-scale discrete-time dynamical systems decomposed into interconnected subsystems, dissipativity of the composite system is shown to be determined from the dissipativity properties of the individual subsystems and the nature of the interconnections. In particular, we develop extended Kalman-Yakubovich-Popov conditions, in terms of the local subsystem dynamics and the interconnection constraints, for characterizing vector dissipativeness via vector storage functions for large-scale discrete-time dynamical systems. Finally, using the concepts of vector dissipativity and vector storage functions as candidate vector Lyapunov functions, we develop feedback interconnection stability results of large-scale discrete-time nonlinear dynamical systems. General stability criteria are given for Lyapunov and asymptotic stability of feedback interconnections of large-scale discrete-time dynamical systems. In the case of vector quadratic supply rates involving net subsystem powers and input-output subsystem energies, these results provide a positivity and small gain theorem for large-scale discrete-time systems predicated on vector Lyapunov functions. 


\section{Mathematical preliminaries}

In this section, we introduce notation, several definitions, and some key results needed for analyzing discrete-time large-scale nonlinear dynamical systems. Let $\mathbb{R}$ denote the set of real numbers, let $\overline{\mathbb{Z}}_{+}$denote the set of nonnegative integers, let $\mathbb{R}^{n}$ denote the set of $n \times 1$ column vectors, let $\mathbb{S}^{n}$ denote the set of $n \times n$ symmetric matrices, let $\mathbb{N}^{n}$ (resp., $\mathbb{P}^{n}$ ) denote the set of $n \times n$ nonnegative (resp., positive) definite matrices, let $(\cdot)^{\mathrm{T}}$ denote transpose, and let $I_{n}$ or $I$ denote the $n \times n$ identity matrix. For $v \in \mathbb{R}^{q}$, we write $v \geq \geq 0$ (resp., $v \gg 0$ ) to indicate that every component of $v$ is nonnegative (resp., positive). In this case we say that $v$ is nonnegative or positive, respectively. Let $\overline{\mathbb{R}}_{+}^{q}$ and $\mathbb{R}_{+}^{q}$ denote the nonnegative and positive orthants of $\mathbb{R}^{q}$; that is, if $v \in \mathbb{R}^{q}$, then $v \in \overline{\mathbb{R}}_{+}^{q}$ and $v \in \mathbb{R}_{+}^{q}$ are equivalent, respectively, to $v \geq \geq 0$ and $v \gg 0$. Finally, we write $\|\cdot\|$ for the Euclidean vector norm, $\operatorname{spec}(M)$ for the spectrum of the square matrix $M, \rho(M)$ for the spectral radius of the square matrix $M, \Delta V(x(k))$ for $V(x(k+1))-V(x(k)), \mathscr{B}_{\varepsilon}(\alpha), \alpha \in \mathbb{R}^{n}, \varepsilon>0$, for the open ball centered at $\alpha$ with radius $\varepsilon$, and $M \geq 0$ (resp., $M>0$ ) to denote the fact that the Hermitian matrix $M$ is nonnegative (resp., positive) definite. The following definition introduces the notion of nonnegative matrices.

Definition 2.1 (see $[3,4,9]$ ). Let $W \in \mathbb{R}^{q \times q}$. The matrix $W$ is nonnegative (resp., positive) if $W_{(i, j)} \geq 0$ (resp., $W_{(i, j)}>0$ ), $i, j=1, \ldots, q$. (In this paper it is important to distinguish between a square nonnegative (resp., positive) matrix and a nonnegative-definite (resp., positive-definite) matrix.)

The following definition introduces the notion of class $\mathcal{W}$ functions involving nondecreasing functions.

Definition 2.2. A function $w=\left[w_{1}, \ldots, w_{q}\right]^{\mathrm{T}}: \mathbb{R}^{q} \rightarrow \mathbb{R}^{q}$ is of class $\mathcal{W}$ if $w_{i}\left(r^{\prime}\right) \leq w_{i}\left(r^{\prime \prime}\right)$, $i=1, \ldots, q$, for all $r^{\prime}, r^{\prime \prime} \in \mathbb{R}^{q}$ such that $r_{j}^{\prime} \leq r_{j}^{\prime \prime}, j=1, \ldots, q$, where $r_{j}$ denotes the $j$ th component of $r$.

Note that if $w(r)=W r$, where $W \in \mathbb{R}^{q \times q}$, then the function $w(\cdot)$ is of class $\mathcal{W}$ if and only if $W$ is nonnegative. The following definition introduces the notion of nonnegative functions [9].

Definition 2.3. Let $w=\left[w_{1}, \ldots, w_{q}\right]^{\mathrm{T}}: \mathscr{V} \rightarrow \mathbb{R}^{q}$, where $\mathscr{V}$ is an open subset of $\mathbb{R}^{q}$ that contains $\overline{\mathbb{R}}_{+}^{q}$. Then $w$ is nonnegative if $w(r) \geq \geq 0$ for all $r \in \overline{\mathbb{R}}_{+}^{q}$.

Note that if $w: \mathbb{R}^{q} \rightarrow \mathbb{R}^{q}$ is such that $w(\cdot) \in \mathcal{W}$ and $w(0) \geq \geq 0$, then $w$ is nonnegative. Note that, if $w(r)=W r$, then $w(\cdot)$ is nonnegative if and only if $W \in \mathbb{R}^{q \times q}$ is nonnegative.

Proposition 2.4 (see [9]). Suppose $\overline{\mathbb{R}}_{+}^{q} \subset \mathcal{V}$. Then $\overline{\mathbb{R}}_{+}^{q}$ is an invariant set with respect to

$$
r(k+1)=w(r(k)), \quad r(0)=r_{0}, \quad k \in \overline{\mathbb{Z}}_{+},
$$

if and only if $w: \mathscr{V} \rightarrow \mathbb{R}^{q}$ is nonnegative.

The following lemma is needed for developing several of the results in later sections. For the statement of this lemma, the following definition is required. 
Definition 2.5. The equilibrium solution $r(k) \equiv r_{\mathrm{e}}$ of (2.1) is Lyapunov stable if, for every $\varepsilon>0$, there exists $\delta=\delta(\varepsilon)>0$ such that if $r_{0} \in \mathscr{B}_{\delta}\left(r_{\mathrm{e}}\right) \cap \overline{\mathbb{R}}_{+}^{q}$, then $r(k) \in \mathscr{B}_{\varepsilon}\left(r_{\mathrm{e}}\right) \cap \overline{\mathbb{R}}_{+}^{q}$, $k \in \overline{\mathbb{Z}}_{+}$. The equilibrium solution $r(k) \equiv r_{\mathrm{e}}$ of (2.1) is semistable if it is Lyapunov stable and there exists $\delta>0$ such that if $r_{0} \in \mathscr{B}_{\delta}\left(r_{\mathrm{e}}\right) \cap \overline{\mathbb{R}}_{+}^{q}$, then $\lim _{k \rightarrow \infty} r(k)$ exists and converges to a Lyapunov stable equilibrium point. The equilibrium solution $r(k) \equiv r_{\mathrm{e}}$ of (2.1) is asymptotically stable if it is Lyapunov stable and there exists $\delta>0$ such that if $r_{0} \in \mathscr{B}_{\delta}\left(r_{\mathrm{e}}\right) \cap \overline{\mathbb{R}}_{+}^{q}$, then $\lim _{k \rightarrow \infty} r(k)=r_{\mathrm{e}}$. Finally, the equilibrium solution $r(k) \equiv r_{\mathrm{e}}$ of (2.1) is globally asymptotically stable if the previous statement holds for all $r_{0} \in \overline{\mathbb{R}}_{+}^{q}$.

Recall that a matrix $W \in \mathbb{R}^{q \times q}$ is semistable if and only if $\lim _{k \rightarrow \infty} W^{k}$ exists [9] while $W$ is asymptotically stable if and only if $\lim _{k \rightarrow \infty} W^{k}=0$.

Lemma 2.6. Suppose $W \in \mathbb{R}^{q \times q}$ is nonsingular and nonnegative. If $W$ is semistable (resp., asymptotically stable), then there exist a scalar $\alpha \geq 1$ (resp., $\alpha>1$ ) and a nonnegative vector $p \in \overline{\mathbb{R}}_{+}^{q}, p \neq 0$, (resp., positive vector $p \in \mathbb{R}_{+}^{q}$ ) such that

$$
W^{-\mathrm{T}} p=\alpha p
$$

Proof. Since $W$ is semistable, it follows from [9, Theorem 3.3] that $|\lambda|<1$ or $\lambda=1$ and $\lambda=1$ is semisimple, where $\lambda \in \operatorname{spec}(W)$. Since $W^{\mathrm{T}} \geq \geq 0$, it follows from the PerronFrobenius theorem that $\rho(W) \in \operatorname{spec}(W)$ and hence there exists $p \geq \geq 0, p \neq 0$, such that $W^{\mathrm{T}} p=\rho(W) p$. In addition, since $W$ is nonsingular, $\rho(W)>0$. Hence, $W^{\mathrm{T}} p=\alpha^{-1} p$, where $\alpha \triangleq 1 / \rho(W)$, which proves that there exist $p \geq \geq 0, p \neq 0$, and $\alpha \geq 1$ such that (2.2) holds. In the case where $W$ is asymptotically stable, the result is a direct consequence of the Perron-Frobenius theorem.

Next, we present a stability result for discrete-time large-scale nonlinear dynamical systems using vector Lyapunov functions. In particular, we consider discrete-time nonlinear dynamical systems of the form

$$
x(k+1)=F(x(k)), \quad x\left(k_{0}\right)=x_{0}, \quad k \geq k_{0},
$$

where $F: \mathscr{D} \rightarrow \mathbb{R}^{n}$ is continuous on $\mathscr{D}, \mathscr{D} \subseteq \mathbb{R}^{n}$ is an open set with $0 \in \mathscr{D}$, and $F(0)=0$. Here, we assume that (2.3) characterizes a discrete-time large-scale nonlinear dynamical system composed of $q$ interconnected subsystems such that, for all $i=1, \ldots, q$, each element of $F(x)$ is given by $F_{i}(x)=f_{i}\left(x_{i}\right)+\mathscr{I}_{i}(x)$, where $f_{i}: \mathbb{R}^{n_{i}} \rightarrow \mathbb{R}^{n_{i}}$ defines the vector field of each isolated subsystem of (2.3), $\mathscr{I}_{i}: \mathscr{D} \rightarrow \mathbb{R}^{n_{i}}$ defines the structure of interconnection dynamics of the $i$ th subsystem with all other subsystems, $x_{i} \in \mathbb{R}^{n_{i}}, f_{i}(0)=0$, $\Phi_{i}(0)=0$, and $\sum_{i=1}^{q} n_{i}=n$. For the discrete-time large-scale nonlinear dynamical system (2.3), we note that the subsystem states $x_{i}(k), k \geq k_{0}$, for all $i=1, \ldots, q$, belong to $\mathbb{R}^{n_{i}}$ as long as $x(k) \triangleq\left[x_{1}^{\mathrm{T}}(k), \ldots, x_{q}^{\mathrm{T}}(k)\right]^{\mathrm{T}} \in \mathscr{D}, k \geq k_{0}$. The next theorem presents a stability result for (2.3) via vector Lyapunov functions by relating the stability properties of a comparison system to the stability properties of the discrete-time large-scale nonlinear dynamical system.

Theorem 2.7 (see [11]). Consider the discrete-time large-scale nonlinear dynamical system given by (2.3). Suppose there exist a continuous vector function $V: \mathscr{D} \rightarrow \overline{\mathbb{R}}_{+}^{q}$ and a positive 
vector $p \in \mathbb{R}_{+}^{q}$ such that $V(0)=0$, the scalar function $v: \mathscr{D} \rightarrow \overline{\mathbb{R}}_{+}$defined by $v(x)=p^{\mathrm{T}} V(x)$, $x \in \mathscr{D}$, is such that $v(0)=0, v(x)>0, x \neq 0$, and

$$
V(F(x)) \leq \leq w(V(x)), \quad x \in \mathscr{D},
$$

where $w: \overline{\mathbb{R}}_{+}^{q} \rightarrow \mathbb{R}^{q}$ is a class $\mathcal{W}$ function such that $w(0)=0$. Then the stability properties of the zero solution $r(k) \equiv 0$ to

$$
r(k+1)=w(r(k)), \quad r\left(k_{0}\right)=r_{0}, \quad k \geq k_{0},
$$

imply the corresponding stability properties of the zero solution $x(k) \equiv 0$ to (2.3). That is, if the zero solution $r(k) \equiv 0$ to (2.5) is Lyapunov (resp., asymptotically) stable, then the zero solution $x(k) \equiv 0$ to (2.3) is Lyapunov (resp., asymptotically) stable. If, in addition, $\mathscr{D}=\mathbb{R}^{n}$ and $V(x) \rightarrow \infty$ as $\|x\| \rightarrow \infty$, then global asymptotic stability of the zero solution $r(k) \equiv 0$ to (2.5) implies global asymptotic stability of the zero solution $x(k) \equiv 0$ to (2.3).

If $V: \mathscr{D} \rightarrow \overline{\mathbb{R}}_{+}^{q}$ satisfies the conditions of Theorem 2.7, we say that $V(x), x \in \mathscr{D}$, is a vector Lyapunov function for the discrete-time large-scale nonlinear dynamical system (2.3). Finally, we recall the notions of dissipativity [6] and geometric dissipativity $[8,9]$ for discrete-time nonlinear dynamical systems $\mathscr{G}$ of the form

$$
\begin{gathered}
x(k+1)=f(x(k))+G(x(k)) u(k), \quad x\left(k_{0}\right)=x_{0}, \quad k \geq k_{0}, \\
y(k)=h(x(k))+J(x(k)) u(k),
\end{gathered}
$$

where $x \in \mathscr{D} \subseteq \mathbb{R}^{n}, u \in \mathscr{U} \subseteq \mathbb{R}^{m}, y \in \mathscr{Y} \subseteq \mathbb{R}^{l}, f: \mathscr{D} \rightarrow \mathbb{R}^{n}$ satisfies $f(0)=0, G: \mathscr{D} \rightarrow$ $\mathbb{R}^{n \times m}, h: \mathscr{D} \rightarrow \mathbb{R}^{l}$ satisfies $h(0)=0$, and $J: \mathscr{D} \rightarrow \mathbb{R}^{l \times m}$. For the discrete-time nonlinear dynamical system $\mathscr{G}$, we assume that the required properties for the existence and uniqueness of solutions are satisfied; that is, $u(\cdot)$ satisfies sufficient regularity conditions such that (2.6) has a unique solution forward in time. Note that since all input-output pairs $u \in \mathcal{U}, y \in \mathcal{Y}$ of the discrete-time nonlinear dynamical system $\mathscr{G}$ are defined on $\overline{\mathbb{Z}}_{+}$, the supply rate [22] satisfying $s(0,0)=0$ is locally summable for all input-output pairs satisfying (2.6), (2.7); that is, for all input-output pairs $u \in \mathcal{U}, y \in \mathcal{Y}$ satisfying (2.6), (2.7), $s(\cdot, \cdot)$ satisfies $\sum_{k=k_{1}}^{k_{2}}|s(u(k), y(k))|<\infty, k_{1}, k_{2} \in \overline{\mathbb{Z}}_{+}$.

Definition 2.8 (see $[6,8]$ ). The discrete-time nonlinear dynamical system $\mathscr{G}$ given by $(2.6)$, (2.7) is geometrically dissipative (resp., dissipative) with respect to the supply rate $s(u, y)$ if there exist a continuous nonnegative-definite function $v_{\mathrm{s}}: \mathbb{R}^{n} \rightarrow \overline{\mathbb{R}}_{+}$, called a storage function, and a scalar $\rho>1$ (resp., $\rho=1$ ) such that $v_{\mathrm{s}}(0)=0$ and the dissipation inequality

$$
\rho^{k_{2}} v_{\mathrm{s}}\left(x\left(k_{2}\right)\right) \leq \rho^{k_{1}} v_{\mathrm{s}}\left(x\left(k_{1}\right)\right)+\sum_{i=k_{1}}^{k_{2}-1} \rho^{i+1} s(u(i), y(i)), \quad k_{2} \geq k_{1},
$$

is satisfied for all $k_{2} \geq k_{1} \geq k_{0}$, where $x(k), k \geq k_{0}$, is the solution to (2.6) with $u \in \mathcal{U}$. The discrete-time nonlinear dynamical system $\mathscr{G}$ given by (2.6), (2.7) is lossless with respect to the supply rate $s(u, y)$ if the dissipation inequality is satisfied as an equality with $\rho=1$ for all $k_{2} \geq k_{1} \geq k_{0}$. 
An equivalent statement for dissipativity of the dynamical system (2.6), (2.7) is

$$
\Delta v_{s}(x(k)) \leq s(u(k), y(k)), \quad k \geq k_{0}, u \in \mathcal{U}, y \in \mathscr{Y} .
$$

Alternatively, an equivalent statement for geometric dissipativity of the dynamical system (2.6), (2.7) is

$$
\rho v_{\mathrm{s}}(x(k+1))-v_{\mathrm{s}}(x(k)) \leq \rho s(u(k), y(k)), \quad k \geq k_{0}, u \in \mathcal{U}, y \in \mathscr{Y} .
$$

\section{Vector dissipativity theory for discrete-time large-scale nonlinear dynamical systems}

In this section, we extend the notion of dissipative dynamical systems to develop the generalized notion of vector dissipativity for discrete-time large-scale nonlinear dynamical systems. We begin by considering discrete-time nonlinear dynamical systems $\mathscr{G}$ of the form

$$
\begin{gathered}
x(k+1)=F(x(k), u(k)), \quad x\left(k_{0}\right)=x_{0}, \quad k \geq k_{0}, \\
y(k)=H(x(k), u(k)),
\end{gathered}
$$

where $x \in \mathscr{D} \subseteq \mathbb{R}^{n}, u \in \mathcal{U} \subseteq \mathbb{R}^{m}, y \in \mathscr{Y} \subseteq \mathbb{R}^{l}, F: \mathscr{D} \times \mathcal{U} \rightarrow \mathbb{R}^{n}, H: \mathscr{D} \times \mathscr{U} \rightarrow \mathscr{Y}$, $\mathscr{D}$ is an open set with $0 \in \mathscr{D}$, and $F(0,0)=0$. Here, we assume that $\mathscr{G}$ represents a discretetime large-scale dynamical system composed of $q$ interconnected controlled subsystems $\mathscr{G}_{i}$ such that, for all $i=1, \ldots, q$,

$$
\begin{aligned}
F_{i}\left(x, u_{i}\right) & =f_{i}\left(x_{i}\right)+\Phi_{i}(x)+G_{i}\left(x_{i}\right) u_{i}, \\
H_{i}\left(x_{i}, u_{i}\right) & =h_{i}\left(x_{i}\right)+J_{i}\left(x_{i}\right) u_{i},
\end{aligned}
$$

where $x_{i} \in \mathbb{R}^{n_{i}}, u_{i} \in \mathcal{U}_{i} \subseteq \mathbb{R}^{m_{i}}, y_{i} \triangleq H_{i}\left(x_{i}, u_{i}\right) \in \mathscr{Y}_{i} \subseteq \mathbb{R}^{l_{i}},\left(u_{i}, y_{i}\right)$ is the input-output pair for the $i$ th subsystem, $f_{i}: \mathbb{R}^{n_{i}} \rightarrow \mathbb{R}^{n_{i}}$ and $\mathscr{I}_{i}: \mathscr{D} \rightarrow \mathbb{R}^{n_{i}}$ are continuous and satisfy $f_{i}(0)=0$ and $\Phi_{i}(0)=0, G_{i}: \mathbb{R}^{n_{i}} \rightarrow \mathbb{R}^{n_{i} \times m_{i}}$ is continuous, $h_{i}: \mathbb{R}^{n_{i}} \rightarrow \mathbb{R}^{l_{i}}$ satisfies $h_{i}(0)=0, J_{i}: \mathbb{R}^{n_{i}} \rightarrow$ $\mathbb{R}^{l_{i} \times m_{i}}, \sum_{i=1}^{q} n_{i}=n, \sum_{i=1}^{q} m_{i}=m$, and $\sum_{i=1}^{q} l_{i}=l$. Furthermore, for the system $\mathscr{G}_{\mathrm{w}}$ we assume that the required properties for the existence and uniqueness of solutions are satisfied. We define the composite input and composite output for the discrete-time largescale system $\mathscr{G}$ as $u \triangleq\left[u_{1}^{\mathrm{T}}, \ldots, u_{q}^{\mathrm{T}}\right]^{\mathrm{T}}$ and $y \triangleq\left[y_{1}^{\mathrm{T}}, \ldots, y_{q}^{\mathrm{T}}\right]^{\mathrm{T}}$, respectively. Note that, in this case, the set $\boldsymbol{u}=\boldsymbol{U}_{1} \times \cdots \times \boldsymbol{U}_{q}$ contains the set of input values and $\mathscr{Y}=\mathscr{Y}_{1} \times \cdots \times \mathscr{Y}_{q}$ contains the set of output values.

Definition 3.1. For the discrete-time large-scale nonlinear dynamical system $\mathscr{G}$ given by (3.1), (3.2), a vector function $S=\left[s_{1}, \ldots, s_{q}\right]^{\mathrm{T}}: \mathcal{U} \times \mathscr{y} \rightarrow \mathbb{R}^{q}$ such that $S(u, y) \triangleq\left[s_{1}\left(u_{1}, y_{1}\right)\right.$, $\left.\ldots, s_{q}\left(u_{q}, y_{q}\right)\right]^{\mathrm{T}}$ and $S(0,0)=0$ is called a vector supply rate.

Note that, since all input-output pairs $\left(u_{i}, y_{i}\right) \in \mathcal{U}_{i} \times \mathscr{Y}_{i}, i=1, \ldots, q$, satisfying (3.1), (3.2) are defined on $\overline{\mathbb{Z}}_{+}, s_{i}(\cdot, \cdot)$ satisfies $\sum_{k=k_{1}}^{k_{2}}\left|s_{i}\left(u_{i}(k), y_{i}(k)\right)\right|<\infty, k_{1}, k_{2} \in \overline{\mathbb{Z}}_{+}$.

Definition 3.2. The discrete-time large-scale nonlinear dynamical system $\varphi_{\text {given }}$ by (3.1), (3.2) is vector dissipative (resp., geometrically vector dissipative) with respect to the vector 
supply rate $S(u, y)$ if there exist a continuous, nonnegative definite vector function $V_{\mathrm{s}}=$ $\left[v_{\mathrm{s} 1}, \ldots, v_{\mathrm{s} q}\right]^{\mathrm{T}}: \mathscr{D} \rightarrow \overline{\mathbb{R}}_{+}^{q}$, called a vector storage function, and a nonsingular nonnegative dissipation matrix $W \in \mathbb{R}^{q \times q}$ such that $V_{s}(0)=0, W$ is semistable (resp., asymptotically stable), and the vector dissipation inequality

$$
V_{s}(x(k)) \leq \leq W^{k-k_{0}} V_{s}\left(x\left(k_{0}\right)\right)+\sum_{i=k_{0}}^{k-1} W^{k-1-i} S(u(i), y(i)), \quad k \geq k_{0},
$$

is satisfied, where $x(k), k \geq k_{0}$, is the solution to (3.1) with $u \in \mathcal{U}$. The discrete-time largescale nonlinear dynamical system $\mathscr{G}$ given by (3.1), (3.2) is vector lossless with respect to the vector supply rate $S(u, y)$ if the vector dissipation inequality is satisfied as an equality with $W$ semistable.

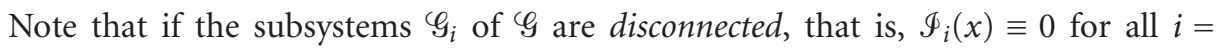
$1, \ldots, q$, and $W \in \mathbb{R}^{q \times q}$ is diagonal, positive definite, and semistable, then it follows from Definition 3.2 that each of the isolated subsystems $\varphi_{i}$ is dissipative or geometrically dissipative in the sense of Definition 2.8. A similar remark holds in the case where $q=1$. Next, define the vector available storage of the discrete-time large-scale nonlinear dynamical system $\mathscr{G}$ by

$$
V_{\mathrm{a}}\left(x_{0}\right) \triangleq \sup _{K \geq k_{0}, u(\cdot)}\left[-\sum_{k=k_{0}}^{K-1} W^{-\left(k+1-k_{0}\right)} S(u(k), y(k))\right],
$$

where $x(k), k \geq k_{0}$, is the solution to (3.1) with $x\left(k_{0}\right)=x_{0}$ and admissible inputs $u \in$ U. The supremum in (3.5) is taken componentwise, which implies that, for different elements of $V_{\mathrm{a}}(\cdot)$, the supremum is calculated separately. Note that $V_{\mathrm{a}}\left(x_{0}\right) \geq \geq 0, x_{0} \in \mathscr{D}$, since $V_{\mathrm{a}}\left(x_{0}\right)$ is the supremum over a set of vectors containing the zero vector $\left(K=k_{0}\right)$. To state the main results of this section, the following definition is required.

Definition 3.3 (see [9]). The discrete-time large-scale nonlinear dynamical system $\mathscr{G}_{\text {given }}$ by (3.1), (3.2) is completely reachable if, for all $x_{0} \in \mathscr{D} \subseteq \mathbb{R}^{n}$, there exist a $k_{\mathrm{i}}<k_{0}$ and a square summable input $u(\cdot)$ defined on $\left[k_{\mathrm{i}}, k_{0}\right]$ such that the state $x(k), k \geq k_{\mathrm{i}}$, can be driven from $x\left(k_{\mathrm{i}}\right)=0$ to $x\left(k_{0}\right)=x_{0}$. A discrete-time large-scale nonlinear dynamical

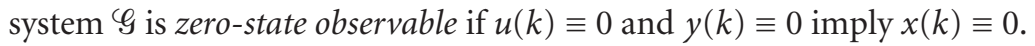

Theorem 3.4. Consider the discrete-time large-scale nonlinear dynamical system $\mathscr{G}$ given by (3.1), (3.2) and assume that $\varphi$ is completely reachable. Let $W \in \mathbb{R}^{q \times q}$ be nonsingular, nonnegative, and semistable (resp., asymptotically stable). Then

$$
\sum_{k=k_{0}}^{K-1} W^{-\left(k+1-k_{0}\right)} S(u(k), y(k)) \geq \geq 0, \quad K \geq k_{0}, u \in \mathcal{U},
$$

for $x\left(k_{0}\right)=0$ if and only if $V_{\mathrm{a}}(0)=0$ and $V_{\mathrm{a}}(x)$ is finite for all $x \in \mathscr{D}$. Moreover, if (3.6) holds, then $V_{\mathrm{a}}(x), x \in \mathscr{D}$, is a vector storage function for $\mathscr{G}$ and hence $\mathscr{G}$ is vector dissipative (resp., geometrically vector dissipative) with respect to the vector supply rate $S(u, y)$. 
44 Vector dissipativity and discrete-time large-scale systems

Proof. Suppose $V_{\mathrm{a}}(0)=0$ and $V_{\mathrm{a}}(x), x \in \mathscr{D}$, is finite. Then

$$
0=V_{\mathrm{a}}(0)=\sup _{K \geq k_{0}, u(\cdot)}\left[-\sum_{k=k_{0}}^{K-1} W^{-\left(k+1-k_{0}\right)} S(u(k), y(k))\right],
$$

which implies (3.6).

Next, suppose (3.6) holds. Then, for $x\left(k_{0}\right)=0$,

$$
\sup _{K \geq k_{0}, u(\cdot)}\left[-\sum_{k=k_{0}}^{K-1} W^{-\left(k+1-k_{0}\right)} S(u(k), y(k))\right] \leq \leq 0
$$

which implies that $V_{\mathrm{a}}(0) \leq \leq 0$. However, since $V_{\mathrm{a}}\left(x_{0}\right) \geq \geq 0, x_{0} \in \mathscr{D}$, it follows that $V_{\mathrm{a}}(0)=$ 0 . Moreover, since $\mathscr{G}$ is completely reachable, it follows that, for every $x_{0} \in \mathscr{D}$, there exists $\hat{k}>k_{0}$ and an admissible input $u(\cdot)$ defined on $\left[k_{0}, \hat{k}\right]$ such that $x(\hat{k})=x_{0}$. Now, since (3.6) holds for $x\left(k_{0}\right)=0$, it follows that, for all admissible $u(\cdot) \in \mathcal{U}$,

$$
\sum_{k=k_{0}}^{K-1} W^{-\left(k+1-k_{0}\right)} S(u(k), y(k)) \geq \geq 0, \quad K \geq \hat{k}
$$

or, equivalently, multiplying (3.9) by the nonnegative matrix $W^{\hat{k}-k_{0}}, \hat{k}>k_{0}$, yields

$$
\begin{aligned}
-\sum_{k=\hat{k}}^{K-1} W^{-(k+1-\hat{k})} S(u(k), y(k)) & \leq \leq \sum_{k=k_{0}}^{\hat{k}-1} W^{-(k+1-\hat{k})} S(u(k), y(k)) \\
& \leq \leq Q\left(x_{0}\right) \ll \infty, \quad K \geq \hat{k}, u \in U,
\end{aligned}
$$

where $Q: \mathscr{D} \rightarrow \mathbb{R}^{q}$. Hence,

$$
V_{\mathrm{a}}\left(x_{0}\right)=\sup _{K \geq \hat{k}, u(\cdot)}\left[-\sum_{k=\hat{k}}^{K-1} W^{-(k+1-\hat{k})} S(u(k), y(k))\right] \leq \leq Q\left(x_{0}\right) \ll \infty, \quad x_{0} \in \mathscr{D},
$$

which implies that $V_{\mathrm{a}}\left(x_{0}\right), x_{0} \in \mathscr{D}$, is finite.

Finally, since (3.6) implies that $V_{\mathrm{a}}(0)=0$ and $V_{\mathrm{a}}(x), x \in \mathscr{D}$, is finite, it follows from the definition of the vector available storage that

$$
\begin{aligned}
-V_{\mathrm{a}}\left(x_{0}\right) \leq & \leq \sum_{k=k_{0}}^{K-1} W^{-\left(k+1-k_{0}\right)} S(u(k), y(k)) \\
= & \sum_{k=k_{0}}^{k_{\mathrm{f}}-1} W^{-\left(k+1-k_{0}\right)} S(u(k), y(k)) \\
& +\sum_{k=k_{\mathrm{f}}}^{K-1} W^{-\left(k+1-k_{0}\right)} S(u(k), y(k)), \quad K \geq k_{0} .
\end{aligned}
$$


Now, multiplying (3.12) by the nonnegative matrix $W^{k_{\mathrm{f}}-k_{0}}, k_{\mathrm{f}}>k_{0}$, it follows that

$$
\begin{aligned}
W^{k_{\mathrm{f}}-k_{0}} & V_{\mathrm{a}}\left(x_{0}\right)+\sum_{k=k_{0}}^{k_{\mathrm{f}}-1} W^{-\left(k+1-k_{\mathrm{f}}\right)} S(u(k), y(k)) \\
\geq & \geq \sup _{K \geq k_{\mathrm{f}}, u(\cdot)}\left[-\sum_{k=k_{\mathrm{f}}}^{K-1} W^{-\left(k+1-k_{\mathrm{f}}\right)} S(u(k), y(k))\right] \\
= & V_{\mathrm{a}}\left(x\left(k_{\mathrm{f}}\right)\right),
\end{aligned}
$$

which implies that $V_{\mathrm{a}}(x), x \in \mathscr{D}$, is a vector storage function and hence $\mathscr{G}$ is vector dissipative (resp., geometrically vector dissipative) with respect to the vector supply rate $S(u, y)$.

It follows from Lemma 2.6 that if $W \in \mathbb{R}^{q \times q}$ is nonsingular, nonnegative, and semistable (resp., asymptotically stable), then there exist a scalar $\alpha \geq 1$ (resp., $\alpha>1$ ) and a nonnegative vector $p \in \overline{\mathbb{R}}_{+}^{q}, p \neq 0$, (resp., $p \in \mathbb{R}_{+}^{q}$ ) such that (2.2) holds. In this case,

$$
p^{\mathrm{T}} W^{-k}=\alpha p^{\mathrm{T}} W^{-(k-1)}=\cdots=\alpha^{k} p^{\mathrm{T}}, \quad k \in \overline{\mathbb{Z}}_{+} .
$$

Using (3.14), we define the (scalar) available storage for the discrete-time large-scale nonlinear dynamical system $\mathscr{G}$ by

$$
\begin{aligned}
v_{\mathrm{a}}\left(x_{0}\right) & \triangleq \sup _{K \geq k_{0}, u(\cdot)}\left[-\sum_{k=k_{0}}^{K-1} p^{\mathrm{T}} W^{-\left(k+1-k_{0}\right)} S(u(k), y(k))\right] \\
& =\sup _{K \geq k_{0}, u(\cdot)}\left[-\sum_{k=k_{0}}^{K-1} \alpha^{k+1-k_{0}} s(u(k), y(k))\right]
\end{aligned}
$$

where $s: \mathcal{U} \times y \rightarrow \mathbb{R}$ defined as $s(u, y) \triangleq p^{\mathrm{T}} S(u, y)$ is the (scalar) supply rate for the discrete-time large-scale nonlinear dynamical system $\mathscr{G}$. Clearly, $v_{\mathrm{a}}(x) \geq 0$ for all $x \in \mathscr{D}$. As in standard dissipativity theory, the available storage $v_{\mathrm{a}}(x), x \in \mathscr{D}$, denotes the maximum amount of (scaled) energy that can be extracted from the discrete-time large-scale nonlinear dynamical system $\mathscr{G}$ at any instant $K$.

The following theorem relates vector storage functions and vector supply rates to scalar storage functions and scalar supply rates of discrete-time large-scale dynamical systems.

Theorem 3.5. Consider the discrete-time large-scale nonlinear dynamical system $G$ given by (3.1), (3.2). Suppose $G$ is vector dissipative (resp., geometrically vector dissipative) with respect to the vector supply rate $S: \mathcal{U} \times \mathscr{Y} \rightarrow \mathbb{R}^{q}$ and with vector storage function $V_{\mathrm{s}}: \mathscr{D} \rightarrow \overline{\mathbb{R}}_{+}^{q}$. Then there exists $p \in \overline{\mathbb{R}}_{+}^{q}, p \neq 0$, (resp., $p \in \mathbb{R}_{+}^{q}$ ) such that $G$ is dissipative (resp., geometrically dissipative) with respect to the scalar supply rate $s(u, y)=p^{\mathrm{T}} S(u, y)$ and with storage function $v_{\mathrm{s}}(x) \triangleq p^{\mathrm{T}} V_{\mathrm{s}}(x), x \in \mathscr{D}$. Moreover, in this case, $v_{\mathrm{a}}(x), x \in \mathscr{D}$, is a storage function for $\mathscr{G}$ and

$$
0 \leq v_{\mathrm{a}}(x) \leq v_{\mathrm{s}}(x), \quad x \in \mathscr{D}
$$


Proof. Suppose $G$ is vector dissipative (resp., geometrically vector dissipative) with respect to the vector supply rate $S(u, y)$. Then there exist a nonsingular, nonnegative, and semistable (resp., asymptotically stable) dissipation matrix $W$ and a vector storage function $V_{s}: \mathscr{D} \rightarrow \overline{\mathbb{R}}_{+}^{q}$ such that the dissipation inequality (3.4) holds. Furthermore, it follows from Lemma 2.6 that there exist $\alpha \geq 1$ (resp., $\alpha>1$ ) and a nonzero vector $p \in \overline{\mathbb{R}}_{+}^{q}$ (resp., $p \in \mathbb{R}_{+}^{q}$ ) satisfying (2.2). Hence, premultiplying (3.4) by $p^{\mathrm{T}}$ and using (3.14), it follows that

$$
v_{\mathrm{s}}(x(k)) \leq \alpha^{-\left(k-k_{0}\right)} v_{\mathrm{s}}\left(x\left(k_{0}\right)\right)+\sum_{i=k_{0}}^{k-1} \alpha^{-(k-1-i)} s(u(i), y(i)), \quad k \geq k_{0}, u \in \mathcal{u}
$$

where $v_{s}(x)=p^{\mathrm{T}} V_{s}(x), x \in \mathscr{D}$, which implies dissipativity (resp., geometric dissipativity) of $\mathscr{G}$ with respect to the supply rate $s(u, y)$ and with storage function $v_{s}(x), x \in \mathscr{D}$. Moreover, since $v_{\mathrm{s}}(0)=0$, it follows from (3.17) that for $x\left(k_{0}\right)=0$,

$$
\sum_{i=k_{0}}^{k-1} \alpha^{i+1-k_{0}} s(u(i), y(i)) \geq 0, \quad k \geq k_{0}, u \in \mathcal{U},
$$

which, using (3.15), implies that $v_{\mathrm{a}}(0)=0$. Now, it can easily be shown that $v_{\mathrm{a}}(x), x \in \mathscr{D}$, satisfies (3.17), and hence the available storage defined by (3.15) is a storage function for G. Finally, it follows from (3.17) that

$$
\begin{aligned}
v_{\mathrm{s}}\left(x\left(k_{0}\right)\right) & \geq \alpha^{k-k_{0}} v_{\mathrm{s}}(x(k))-\sum_{i=k_{0}}^{k-1} \alpha^{i+1-k_{0}} s(u(i), y(i)) \\
& \geq-\sum_{i=k_{0}}^{k-1} \alpha^{i+1-k_{0}} s(u(i), y(i)), \quad k \geq k_{0}, u \in \mathcal{U},
\end{aligned}
$$

which implies that

$$
v_{\mathrm{s}}\left(x\left(k_{0}\right)\right) \geq \sup _{k \geq k_{0}, u(\cdot)}\left[-\sum_{i=k_{0}}^{k-1} \alpha^{i+1-k_{0}} s(u(i), y(i))\right]=v_{\mathrm{a}}\left(x\left(k_{0}\right)\right),
$$

and hence (3.16) holds.

Remark 3.6. It follows from Theorem 3.4 that if (3.6) holds for $x\left(k_{0}\right)=0$, then the vector available storage $V_{\mathrm{a}}(x), x \in \mathscr{D}$, is a vector storage function for $\mathscr{G}$. In this case, it follows from Theorem 3.5 that there exists $p \in \overline{\mathbb{R}}_{+}^{q}, p \neq 0$, such that $v_{\mathrm{s}}(x) \triangleq p^{\mathrm{T}} V_{\mathrm{a}}(x)$ is a storage function for $\mathscr{G}$ that satisfies (3.17), and hence, by (3.16), $v_{\mathrm{a}}(x) \leq p^{\mathrm{T}} V_{\mathrm{a}}(x), x \in \mathscr{D}$.

Remark 3.7. It is important to note that it follows from Theorem 3.5 that if $\mathscr{G}$ is vector dissipative, then $\mathscr{G}$ can either be (scalar) dissipative or (scalar) geometrically dissipative.

The following theorem provides sufficient conditions guaranteeing that all scalar storage functions defined in terms of vector storage functions, that is, $v_{s}(x)=p^{\mathrm{T}} V_{s}(x)$, of a given vector dissipative discrete-time large-scale nonlinear dynamical system are positive definite. 
Theorem 3.8. Consider the discrete-time large-scale nonlinear dynamical system $\mathscr{G}$ given by (3.1), (3.2) and assume that $G$ is zero-state observable. Furthermore, assume that $G$ is vector dissipative (resp., geometrically vector dissipative) with respect to the vector supply rate $S(u, y)$ and there exist $\alpha \geq 1$ and $p \in \mathbb{R}_{+}^{q}$ such that (2.2) holds. In addition, assume that there exist functions $\kappa_{i}: \mathscr{Y}_{i} \rightarrow U_{i}$ such that $\kappa_{i}(0)=0$ and $s_{i}\left(\kappa_{i}\left(y_{i}\right), y_{i}\right)<0, y_{i} \neq 0$, for all $i=1, \ldots, q$. Then, for all vector storage functions $V_{\mathrm{s}}: \mathscr{D} \rightarrow \overline{\mathbb{R}}_{+}^{q}$, the storage function $v_{\mathrm{s}}(x) \triangleq$ $p^{\mathrm{T}} V_{\mathrm{s}}(x), x \in \mathscr{D}$, is positive definite; that is, $v_{\mathrm{s}}(0)=0$ and $v_{\mathrm{s}}(x)>0, x \in \mathscr{D}, x \neq 0$.

Proof. It follows from Theorem 3.5 that $v_{\mathrm{a}}(x), x \in \mathscr{D}$, is a storage function for $\mathscr{G}$ that satisfies (3.17). Next, suppose, ad absurdum, there exists $x \in \mathscr{D}$ such that $v_{\mathrm{a}}(x)=0, x \neq 0$. Then it follows from the definition of $v_{\mathrm{a}}(x), x \in \mathscr{D}$, that for $x\left(k_{0}\right)=x$,

$$
\sum_{k=k_{0}}^{K-1} \alpha^{k+1-k_{0}} s(u(k), y(k)) \geq 0, \quad K \geq k_{0}, u \in \mathcal{U} .
$$

However, for $u_{i}=k_{i}\left(y_{i}\right)$, we have $s_{i}\left(\kappa_{i}\left(y_{i}\right), y_{i}\right)<0, y_{i} \neq 0$, for all $i=1, \ldots, q$, and since $p \gg 0$, it follows that $y_{i}(k)=0, k \geq k_{0}, i=1, \ldots, q$, which further implies that $u_{i}(k)=0$, $k \geq k_{0}, i=1, \ldots, q$. Since $\mathscr{G}$ is zero-state observable, it follows that $x=0$ and hence $v_{\mathrm{a}}(x)=$ 0 if and only if $x=0$. The result now follows from (3.16). Finally, for the geometrically vector dissipative case, it follows from Lemma 2.6 that $p \gg 0$ with the rest of the proof being identical as above.

Next, we introduce the concept of vector required supply of a discrete-time large-scale nonlinear dynamical system. Specifically, define the vector required supply of the discretetime large-scale dynamical system $\mathscr{G}$ by

$$
V_{\mathrm{r}}\left(x_{0}\right) \triangleq \inf _{K \geq-k_{0}+1, u(\cdot)} \sum_{k=-K}^{k_{0}-1} W^{-\left(k+1-k_{0}\right)} S(u(k), y(k)),
$$

where $x(k), k \geq-K$, is the solution to (3.1) with $x(-K)=0$ and $x\left(k_{0}\right)=x_{0}$. Note that since, with $x\left(k_{0}\right)=0$, the infimum in (3.22) is the zero vector, it follows that $V_{\mathrm{r}}(0)=0$. Moreover, since $\mathscr{G}$ is completely reachable, it follows that $V_{\mathrm{r}}(x) \ll \infty, x \in \mathscr{D}$. Using the notion of the vector required supply, we present necessary and sufficient conditions for dissipativity of a large-scale dynamical system with respect to a vector supply rate.

Theorem 3.9. Consider the discrete-time large-scale nonlinear dynamical system $\mathscr{G}$ given by (3.1), (3.2) and assume that $\varphi$ is completely reachable. Then $\varphi$ is vector dissipative (resp., geometrically vector dissipative) with respect to the vector supply rate $S(u, y)$ if and only if

$$
0 \leq \leq V_{\mathrm{r}}(x) \ll \infty, \quad x \in \mathscr{D}
$$

Moreover, if (3.23) holds, then $V_{\mathrm{r}}(x), x \in \mathscr{D}$, is a vector storage function for $G$. Finally, if the vector available storage $V_{\mathrm{a}}(x), x \in \mathscr{D}$, is a vector storage function for $\mathscr{G}$, then

$$
0 \leq \leq V_{\mathrm{a}}(x) \leq \leq V_{\mathrm{r}}(x) \ll \infty, \quad x \in \mathscr{D} .
$$


Proof. Suppose (3.23) holds and let $x(k), k \in \overline{\mathbb{Z}}_{+}$, satisfy (3.1) with admissible inputs $u(k) \in \mathcal{U}, k \in \overline{\mathbb{Z}}_{+}$, and $x\left(k_{0}\right)=x_{0}$. Then it follows from the definition of $V_{\mathrm{r}}(\cdot)$ that for $-K \leq k_{\mathrm{f}} \leq k_{0}-1$ and $u(\cdot) \in \mathcal{U}$,

$$
\begin{aligned}
V_{\mathrm{r}}\left(x_{0}\right) & \leq \leq \sum_{k=-K}^{k_{0}-1} W^{-\left(k+1-k_{0}\right)} S(u(k), y(k)) \\
& =\sum_{k=-K}^{k_{\mathrm{f}}-1} W^{-\left(k+1-k_{0}\right)} S(u(k), y(k))+\sum_{k=k_{\mathrm{f}}}^{k_{0}-1} W^{-\left(k+1-k_{0}\right)} S(u(k), y(k)),
\end{aligned}
$$

and hence,

$$
\begin{aligned}
V_{\mathrm{r}}\left(x_{0}\right) \leq \leq & W^{k_{0}-k_{\mathrm{f}}} \inf _{K \geq-k_{\mathrm{f}}+1, u(\cdot)}\left[\sum_{k=-K}^{k_{\mathrm{f}}-1} W^{-\left(k+1-k_{\mathrm{f}}\right)} S(u(k), y(k))\right] \\
& +\sum_{k=k_{\mathrm{f}}}^{k_{0}-1} W^{-\left(k+1-k_{0}\right)} S(u(k), y(k)) \\
= & W^{k_{0}-k_{\mathrm{f}}} V_{\mathrm{r}}\left(x\left(k_{\mathrm{f}}\right)\right)+\sum_{k=k_{\mathrm{f}}}^{k_{0}-1} W^{k_{0}-1-k} S(u(k), y(k)),
\end{aligned}
$$

which shows that $V_{\mathrm{r}}(x), x \in \mathscr{D}$, is a vector storage function for $\mathscr{G}$ and hence $\mathscr{G}$ is vector dissipative with respect to the vector supply rate $S(u, y)$.

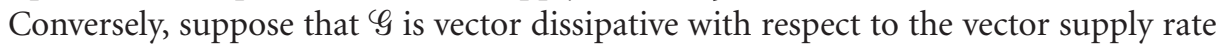
$S(u, y)$. Then there exists a nonnegative vector storage function $V_{s}(x), x \in \mathscr{D}$, such that $V_{s}(0)=0$. Since $\mathscr{G}$ is completely reachable, it follows that for $x\left(k_{0}\right)=x_{0}$, there exist $K>-k_{0}$ and $u(k), k \in\left[-K, k_{0}\right]$, such that $x(-K)=0$. Hence, it follows from the vector dissipation inequality (3.4) that

$$
0 \leq \leq V_{s}\left(x\left(k_{0}\right)\right) \leq \leq W^{k_{0}+K} V_{s}(x(-K))+\sum_{k=-K}^{k_{0}-1} W^{k_{0}-1-k} S(u(k), y(k)),
$$

which implies that for all $K \geq-k_{0}+1$ and $u \in \mathcal{U}$,

$$
0 \leq \leq \sum_{k=-K}^{k_{0}-1} W^{-\left(k+1-k_{0}\right)} S(u(k), y(k))
$$

or, equivalently,

$$
0 \leq \leq \inf _{K \geq-k_{0}+1, u(\cdot)} \sum_{k=-K}^{k_{0}-1} W^{-\left(k+1-k_{0}\right)} S(u(k), y(k))=V_{\mathrm{r}}\left(x_{0}\right) .
$$

Since, by complete reachability, $V_{\mathrm{r}}(x) \ll \infty, x \in \mathscr{D}$, it follows that (3.23) holds. 
Finally, suppose that $V_{\mathrm{a}}(x), x \in \mathscr{D}$, is a vector storage function. Then, for $x(-K)=0$, $x\left(k_{0}\right)=x_{0}$, and $u \in \mathcal{U}$, it follows that

$$
V_{\mathrm{a}}\left(x\left(k_{0}\right)\right) \leq \leq W^{k_{0}+K} V_{\mathrm{a}}(x(-K))+\sum_{k=-K}^{k_{0}-1} W^{k_{0}-1-k} S(u(k), y(k)),
$$

which implies that

$$
0 \leq \leq V_{\mathrm{a}}\left(x\left(k_{0}\right)\right) \leq \leq \inf _{K \geq-k_{0}+1, u(\cdot)} \sum_{k=-K}^{k_{0}-1} W^{-\left(k+1-k_{0}\right)} S(u(k), y(k))=V_{\mathrm{r}}\left(x\left(k_{0}\right)\right), \quad x \in \mathscr{D}
$$

Since $x\left(k_{0}\right)=x_{0} \in \mathscr{D}$ is arbitrary and, by complete reachability, $V_{\mathrm{r}}(x) \ll \infty, x \in \mathscr{D},(3.31)$ implies (3.24).

The next result is a direct consequence of Theorems 3.4 and 3.9.

Proposition 3.10. Consider the discrete-time large-scale nonlinear dynamical system $\mathscr{G}$ given by (3.1), (3.2). Let $M=\operatorname{diag}\left[\mu_{1}, \ldots, \mu_{q}\right]$ be such that $0 \leq \mu_{i} \leq 1, i=1, \ldots$, q. If $V_{\mathrm{a}}(x)$, $x \in \mathscr{D}$, and $V_{\mathrm{r}}(x), x \in \mathscr{D}$, are vector storage functions for $\mathscr{G}$, then

$$
V_{\mathrm{s}}(x)=M V_{\mathrm{a}}(x)+\left(I_{q}-M\right) V_{\mathrm{r}}(x), \quad x \in \mathscr{D},
$$

is a vector storage function for $\mathscr{G}$.

Proof. First note that $M \geq \geq 0$ and $I_{q}-M \geq \geq 0$ if and only if $M=\operatorname{diag}\left[\mu_{1}, \ldots, \mu_{q}\right]$ and $\mu_{i} \in[0,1], i=1, \ldots, q$. Now, the result is a direct consequence of the vector dissipation inequality (3.4) by noting that if $V_{\mathrm{a}}(x)$ and $V_{\mathrm{r}}(x)$ satisfy (3.4), then $V_{\mathrm{s}}(x)$ satisfies (3.4).

Next, recall that if $\varphi$ is vector dissipative (resp., geometrically vector dissipative), then there exist $p \in \overline{\mathbb{R}}_{+}^{q}, p \neq 0$, and $\alpha \geq 1$ (resp., $p \in \mathbb{R}_{+}^{q}$ and $\alpha>1$ ) such that (2.2) and (3.14) hold. Now, define the (scalar) required supply for the large-scale nonlinear dynamical system $\mathscr{G}$ by

$$
\begin{aligned}
v_{\mathrm{r}}\left(x_{0}\right) & \triangleq \inf _{K \geq-k_{0}+1, u(\cdot)} \sum_{k=-K}^{k_{0}-1} p^{\mathrm{T}} W^{-\left(k+1-k_{0}\right)} S(u(k), y(k)) \\
& =\inf _{K \geq-k_{0}+1, u(\cdot)} \sum_{k=-K}^{k_{0}-1} \alpha^{k+1-k_{0}} s(u(k), y(k)), \quad x_{0} \in \mathscr{D},
\end{aligned}
$$

where $s(u, y)=p^{\mathrm{T}} S(u, y)$ and $x(k), k \geq-K$, is the solution to (3.1) with $x(-K)=0$ and $x\left(k_{0}\right)=x_{0}$. It follows from (3.33) that the required supply of a discrete-time large-scale nonlinear dynamical system is the minimum amount of generalized energy which can be delivered to the discrete-time large-scale system in order to transfer it from an initial state $x(-K)=0$ to a given state $x\left(k_{0}\right)=x_{0}$. Using the same arguments as in case of the vector required supply, it follows that $v_{\mathrm{r}}(0)=0$ and $v_{\mathrm{r}}(x)<\infty, x \in \mathscr{D}$. 
Next, using the notion of required supply, we show that all storage functions of the form $v_{\mathrm{s}}(x)=p^{\mathrm{T}} V_{\mathrm{s}}(x)$, where $p \in \overline{\mathbb{R}}_{+}^{q}, p \neq 0$, are bounded from above by the required supply and bounded from below by the available storage. Hence, a dissipative discrete-time large-scale nonlinear dynamical system can only deliver to its surroundings a fraction of all of its stored subsystem energies and can only store a fraction of the work done to all of its subsystems.

CoRollary 3.11. Consider the discrete-time large-scale nonlinear dynamical system 9 given by (3.1), (3.2). Assume that $G$ is vector dissipative with respect to a vector supply rate $S(u, y)$ and with vector storage function $V_{\mathrm{s}}: \mathscr{D} \rightarrow \overline{\mathbb{R}}_{+}^{q}$. Then $v_{\mathrm{r}}(x), x \in \mathscr{D}$, is a storage function for $\mathscr{G}$. Moreover, if $v_{\mathrm{s}}(x) \triangleq p^{\mathrm{T}} V_{\mathrm{s}}(x), x \in \mathscr{D}$, where $p \in \overline{\mathbb{R}}_{+}^{q}, p \neq 0$, then

$$
0 \leq v_{\mathrm{a}}(x) \leq v_{\mathrm{s}}(x) \leq v_{\mathrm{r}}(x)<\infty, \quad x \in \mathscr{D} .
$$

Proof. It follows from Theorem 3.5 that if $\mathscr{G}$ is vector dissipative with respect to the vector supply rate $S(u, y)$ and with a vector storage function $V_{\mathrm{s}}: \mathscr{D} \rightarrow \overline{\mathbb{R}}_{+}^{q}$, then there exists $p \in$ $\overline{\mathbb{R}}_{+}^{q}, p \neq 0$, such that $G$ is dissipative with respect to the supply rate $s(u, y)=p^{\mathrm{T}} S(u, y)$ and with storage function $v_{\mathrm{s}}(x)=p^{\mathrm{T}} V_{\mathrm{s}}(x), x \in \mathscr{D}$. Hence, it follows from (3.17), with $x(-K)=0$ and $x\left(k_{0}\right)=x_{0}$, that

$$
\sum_{k=-K}^{k_{0}-1} \alpha^{k+1-k_{0}} s(u(k), y(k)) \geq 0, \quad K \geq-k_{0}, u \in \mathcal{U},
$$

which implies that $v_{\mathrm{r}}\left(x_{0}\right) \geq 0, x_{0} \in \mathscr{D}$. Furthermore, it is easy to see from the definition of a required supply that $v_{\mathrm{r}}(x), x \in \mathscr{D}$, satisfies the dissipation inequality (3.17). Hence, $v_{\mathrm{r}}(x), x \in \mathscr{D}$, is a storage function for $\mathscr{G}$. Moreover, it follows from the dissipation inequality (3.17), with $x(-K)=0, x\left(k_{0}\right)=x_{0}$, and $u \in \mathcal{U}$, that

$$
\begin{aligned}
\alpha^{k_{0}} v_{\mathrm{s}}\left(x\left(k_{0}\right)\right) & \leq \alpha^{-K} v_{\mathrm{s}}(x(-K))+\sum_{k=-K}^{k_{0}-1} \alpha^{k+1} s(u(k), y(k)) \\
& =\sum_{k=-K}^{k_{0}-1} \alpha^{k+1} s(u(k), y(k)),
\end{aligned}
$$

which implies that

$$
v_{\mathrm{s}}\left(x\left(k_{0}\right)\right) \leq \inf _{K \geq-k_{0}+1, u(\cdot)} \sum_{k=-K}^{k_{0}-1} \alpha^{k+1-k_{0}} s(u(k), y(k))=v_{\mathrm{r}}\left(x\left(k_{0}\right)\right) .
$$

Finally, it follows from Theorem 3.5 that $v_{\mathrm{a}}(x), x \in \mathscr{D}$, is a storage function for $\mathscr{G}$, and hence, using (3.16) and (3.37), (3.34) holds.

Remark 3.12. It follows from Theorem 3.9 that if $\mathscr{G}$ is vector dissipative with respect to the vector supply rate $S(u, y)$, then $V_{\mathrm{r}}(x), x \in \mathscr{D}$, is a vector storage function for $\mathscr{G}$ and, 
by Theorem 3.5, there exists $p \in \overline{\mathbb{R}}_{+}^{q}, p \neq 0$, such that $v_{\mathrm{s}}(x) \triangleq p^{\mathrm{T}} V_{\mathrm{r}}(x), x \in \mathscr{D}$, is a storage function for $\mathscr{G}$ satisfying (3.17). Hence, it follows from Corollary 3.11 that $p^{\mathrm{T}} V_{\mathrm{r}}(x) \leq$ $v_{\mathrm{r}}(x), x \in \mathscr{D}$.

The next result relates vector (resp., scalar) available storage and vector (resp., scalar) required supply for vector lossless discrete-time large-scale dynamical systems.

Theorem 3.13. Consider the discrete-time large-scale nonlinear dynamical system $G$ given by (3.1), (3.2). Assume that $G$ is completely reachable to and from the origin. If $G$ is vector lossless with respect to the vector supply rate $S(u, y)$ and $V_{\mathrm{a}}(x), x \in \mathscr{D}$, is a vector storage function, then $V_{\mathrm{a}}(x)=V_{\mathrm{r}}(x), x \in \mathscr{D}$. Moreover, if $V_{\mathrm{s}}(x), x \in \mathscr{D}$, is a vector storage function, then all (scalar) storage functions of the form $v_{s}(x)=p^{\mathrm{T}} V_{\mathrm{s}}(x), x \in \mathscr{D}$, where $p \in \overline{\mathbb{R}}_{+}^{q}, p \neq 0$, are given by

$$
v_{\mathrm{s}}\left(x_{0}\right)=v_{\mathrm{a}}\left(x_{0}\right)=v_{\mathrm{r}}\left(x_{0}\right)=-\sum_{k=k_{0}}^{K-1} \alpha^{k+1-k_{0}} s(u(k), y(k))=\sum_{k=-K}^{k_{0}-1} \alpha^{k+1-k_{0}} s(u(k), y(k)),
$$

where $x(k), k \geq k_{0}$, is the solution to (3.1) with $u \in U, x(-K)=0, x(K)=0, x\left(k_{0}\right)=x_{0} \in$ $\mathscr{D}$, and $s(u, y)=p^{\mathrm{T}} S(u, y)$.

Proof. Suppose $\mathscr{G}$ is vector lossless with respect to the vector supply rate $S(u, y)$. Since $\mathscr{G}$ is completely reachable to and from the origin, it follows that, for every $x_{0}=x\left(k_{0}\right) \in$ $\mathscr{D}$, there exist $K_{+}>k_{0},-K_{-}<k_{0}$, and $u(k) \in \mathscr{U}, k \in\left[-K_{-}, K_{+}\right]$, such that $x\left(-K_{-}\right)=0$, $x\left(K_{+}\right)=0$, and $x\left(k_{0}\right)=x_{0}$. Now, it follows from the dissipation inequality (3.4) which is satisfied as an equality that

$$
0=\sum_{k=-K_{-}}^{K_{+}-1} W^{K_{+}-1-k} S(u(k), y(k))
$$

or, equivalently,

$$
\begin{aligned}
0 & =\sum_{k=-K_{-}}^{K_{+}-1} W^{-\left(k+1-k_{0}\right)} S(u(k), y(k)) \\
& =\sum_{k=-K_{-}}^{k_{0}-1} W^{-\left(k+1-k_{0}\right)} S(u(k), y(k))+\sum_{k=k_{0}}^{K_{+}-1} W^{-\left(k+1-k_{0}\right)} S(u(k), y(k)) \\
& \geq \geq \inf _{K \geq-k_{0}+1, u(\cdot)} \sum_{k=-K}^{k_{0}-1} W^{-\left(k+1-k_{0}\right)} S(u(k), y(k))+\inf _{K \geq k_{0}, u(\cdot)} \sum_{k=k_{0}}^{K-1} W^{-\left(k+1-k_{0}\right)} S(u(k), y(k)) \\
& =V_{\mathrm{r}}\left(x_{0}\right)-V_{\mathrm{a}}\left(x_{0}\right),
\end{aligned}
$$

which implies that $V_{\mathrm{r}}\left(x_{0}\right) \leq \leq V_{\mathrm{a}}\left(x_{0}\right), x_{0} \in \mathscr{D}$. However, it follows from Theorem 3.9 that if $\mathscr{G}$ is vector dissipative and $V_{\mathrm{a}}(x), x \in \mathscr{D}$, is a vector storage function, then $V_{\mathrm{a}}(x) \leq \leq V_{\mathrm{r}}(x)$, $x \in \mathscr{D}$, which along with (3.40) implies that $V_{\mathrm{a}}(x)=V_{\mathrm{r}}(x), x \in \mathscr{D}$. Furthermore, since $\mathscr{G}$ 
is vector lossless, there exist a nonzero vector $p \in \overline{\mathbb{R}}_{+}^{q}$ and a scalar $\alpha \geq 0$ satisfying (2.2). Now, it follows from (3.39) that

$$
\begin{aligned}
0 & =\sum_{k=-K_{-}}^{K_{+}-1} p^{\mathrm{T}} W^{-\left(k+1-k_{0}\right)} S(u(k), y(k))=\sum_{k=-K_{-}}^{K_{+}-1} \alpha^{k+1-k_{0}} s(u(k), y(k)) \\
& =\sum_{k=-K_{-}}^{k_{0}-1} \alpha^{k+1-k_{0}} s(u(k), y(k))+\sum_{k=k_{0}}^{K_{+}-1} \alpha^{k+1-k_{0}} s(u(k), y(k)) \\
& \geq \inf _{K \geq-k_{0}+1, u(\cdot)} \sum_{k=-K}^{k_{0}-1} \alpha^{k+1-k_{0}} s(u(k), y(k))+\inf _{K \geq k_{0}, u(\cdot)} \sum_{k=k_{0}}^{K-1} \alpha^{k+1-k_{0}} s(u(k), y(k)) \\
& =v_{\mathrm{r}}\left(x_{0}\right)-v_{\mathrm{a}}\left(x_{0}\right), \quad x_{0} \in \mathscr{D},
\end{aligned}
$$

which along with (3.34) implies that for any (scalar) storage function of the form $v_{\mathrm{s}}(x)=$ $p^{\mathrm{T}} V_{\mathrm{s}}(x), x \in \mathscr{D}$, the equality $v_{\mathrm{a}}(x)=v_{\mathrm{s}}(x)=v_{\mathrm{r}}(x), x \in \mathscr{D}$, holds. Moreover, since $\mathscr{G}$ is vector lossless, the inequalities (3.17) and (3.36) are satisfied as equalities and

$$
v_{\mathrm{s}}\left(x_{0}\right)=-\sum_{k=k_{0}}^{K-1} \alpha^{k+1-k_{0}} s(u(k), y(k))=\sum_{k=-K}^{k_{0}-1} \alpha^{k+1-k_{0}} s(u(k), y(k)),
$$

where $x(k), k \geq k_{0}$, is the solution to (3.1) with $u \in \mathcal{U}, x(-K)=0, x(K)=0$, and $x\left(k_{0}\right)=$ $x_{0} \in \mathscr{D}$.

The next proposition presents a characterization for vector dissipativity of discretetime large-scale nonlinear dynamical systems.

Proposition 3.14. Consider the discrete-time large-scale nonlinear dynamical system $\mathscr{G}$ given by (3.1), (3.2) and assume $V_{\mathrm{s}}=\left[v_{\mathrm{s} 1}, \ldots, v_{\mathrm{s} q}\right]^{\mathrm{T}}: \mathscr{D} \rightarrow \overline{\mathbb{R}}_{+}^{q}$ is a continuous vector storage function for $G$. Then $G$ is vector dissipative with respect to the vector supply rate $S(u, y)$ if and only if

$$
V_{\mathrm{s}}(x(k+1)) \leq \leq W V_{\mathrm{s}}(x(k))+S(u(k), y(k)), \quad k \geq k_{0}, u \in \mathcal{U} .
$$

Proof. The proof is immediate from (3.4) and hence is omitted.

As a special case of vector dissipativity theory, we can analyze the stability of discretetime large-scale nonlinear dynamical systems. Specifically, assume that the discrete-time large-scale dynamical system $\mathscr{G}$ is vector dissipative (resp., geometrically vector dissipative) with respect to the vector supply rate $S(u, y)$ and with a continuous vector storage function $V_{\mathrm{s}}: \mathscr{D} \rightarrow \overline{\mathbb{R}}_{+}^{q}$. Moreover, assume that the conditions of Theorem 3.8 are satisfied. Then it follows from Proposition 3.14, with $u(k) \equiv 0$ and $y(k) \equiv 0$, that

$$
V_{\mathrm{s}}(x(k+1)) \leq \leq W V_{\mathrm{s}}(x(k)), \quad k \geq k_{0},
$$

where $x(k), k \geq k_{0}$, is a solution to (3.1) with $x\left(k_{0}\right)=x_{0}$ and $u(k) \equiv 0$. Now, it follows from Theorem 2.7, with $w(r)=W r$, that the zero solution $x(k) \equiv 0$ to (3.1), with $u(k) \equiv$ 0 , is Lyapunov (resp., asymptotically) stable. 
More generally, the problem of control system design for discrete-time large-scale nonlinear dynamical systems can be addressed within the framework of vector dissipativity theory. In particular, suppose that there exists a continuous vector function $V_{\mathrm{s}}: \mathscr{D} \rightarrow \overline{\mathbb{R}}_{+}^{q}$ such that $V_{s}(0)=0$ and

$$
V_{s}(x(k+1)) \leq \leq \mathscr{F}\left(V_{s}(x(k)), u(k)\right), \quad k \geq k_{0}, u \in \mathcal{U},
$$

where $\mathscr{F}_{F}: \overline{\mathbb{R}}_{+}^{q} \times \mathbb{R}^{m} \rightarrow \mathbb{R}^{q}$ and $\mathscr{F}(0,0)=0$. Then the control system design problem for a discrete-time large-scale dynamical system reduces to constructing an energy feedback control law $\phi: \overline{\mathbb{R}}_{+}^{q} \rightarrow \mathcal{U}$ of the form

$$
u=\phi\left(V_{\mathrm{s}}(x)\right) \triangleq\left[\phi_{1}^{\mathrm{T}}\left(V_{\mathrm{s}}(x)\right), \ldots, \phi_{q}^{\mathrm{T}}\left(V_{\mathrm{s}}(x)\right)\right]^{\mathrm{T}}, \quad x \in \mathscr{D},
$$

where $\phi_{i}: \overline{\mathbb{R}}_{+}^{q} \rightarrow U_{i}, \phi_{i}(0)=0, i=1, \ldots, q$, such that the zero solution $r(k) \equiv 0$ to the comparison system

$$
r(k+1)=w(r(k)), \quad r\left(k_{0}\right)=V_{\mathrm{s}}\left(x\left(k_{0}\right)\right), \quad k \geq k_{0},
$$

is rendered asymptotically stable, where $w(r) \triangleq \mathscr{F}(r, \phi(r))$ is of class $\mathcal{W}$. In this case, if there exists $p \in \mathbb{R}_{+}^{q}$ such that $v_{\mathrm{s}}(x) \triangleq p^{\mathrm{T}} V_{\mathrm{s}}(x), x \in \mathscr{D}$, is positive definite, then it follows from Theorem 2.7 that the zero solution $x(k) \equiv 0$ to (3.1), with $u$ given by (3.46), is asymptotically stable.

As can be seen from the above discussion, using an energy feedback control architecture and exploiting the comparison system within the control design for discrete-time large-scale nonlinear dynamical systems can significantly reduce the dimensionality of a control synthesis problem in terms of a number of states that need to be stabilized. It should be noted however that, for stability analysis of discrete-time large-scale dynamical systems, the comparison system need not be linear as implied by (3.44). A discrete-time nonlinear comparison system would still guarantee stability of a discrete-time large-scale dynamical system provided that the conditions of Theorem 2.7 are satisfied.

\section{Extended Kalman-Yakubovich-Popov conditions for discrete-time large-scale non- linear dynamical systems}

In this section, we show that vector dissipativeness (resp., geometric vector dissipativeness) of a discrete-time large-scale nonlinear dynamical system $\mathscr{G}$ of the form (3.1), (3.2) can be characterized in terms of the local subsystem functions $f_{i}(\cdot), G_{i}(\cdot), h_{i}(\cdot)$, and $J_{i}(\cdot)$, along with the interconnection structures $\mathscr{I}_{i}(\cdot)$ for $i=1, \ldots, q$. For the results in this section, we consider the special case of dissipative systems with quadratic vector supply rates and set $\mathscr{D}=\mathbb{R}^{n}, \boldsymbol{U}_{i}=\mathbb{R}^{m_{i}}$, and $\mathscr{Y}_{i}=\mathbb{R}^{l_{i}}$. Specifically, let $R_{i} \in \mathbb{S}^{m_{i}}, S_{i} \in \mathbb{R}^{l_{i} \times m_{i}}$, and $Q_{i} \in \mathbb{S}^{l_{i}}$ be given and assume $S(u, y)$ is such that $s_{i}\left(u_{i}, y_{i}\right)=y_{i}^{\mathrm{T}} Q_{i} y_{i}+2 y_{i}^{\mathrm{T}} S_{i} u_{i}+u_{i}^{\mathrm{T}} R_{i} u_{i}, i=$ $1, \ldots, q$. For the statement of the next result, recall that $x=\left[x_{1}^{\mathrm{T}}, \ldots, x_{q}^{\mathrm{T}}\right]^{\mathrm{T}}, u=\left[u_{1}^{\mathrm{T}}, \ldots, u_{q}^{\mathrm{T}}\right]^{\mathrm{T}}$, $y=\left[y_{1}^{\mathrm{T}}, \ldots, y_{q}^{\mathrm{T}}\right]^{\mathrm{T}}, x_{i} \in \mathbb{R}^{n_{i}}, u_{i} \in \mathbb{R}^{m_{i}}, y_{i} \in \mathbb{R}^{l_{i}}, i=1, \ldots, q, \sum_{i=1}^{q} n_{i}=n, \sum_{i=1}^{q} m_{i}=m$, and 
54 Vector dissipativity and discrete-time large-scale systems

$\sum_{i=1}^{q} l_{i}=l$. Furthermore, for (3.1), (3.2), define $\mathscr{F}_{\mathbb{F}}: \mathbb{R}^{n} \rightarrow \mathbb{R}^{n}, G: \mathbb{R}^{n} \rightarrow \mathbb{R}^{n \times m}, h: \mathbb{R}^{n} \rightarrow$ $\mathbb{R}^{l}$, and $J: \mathbb{R}^{n} \rightarrow \mathbb{R}^{l \times m}$ by $\mathscr{F}(x) \triangleq\left[\mathscr{F}_{1}^{\mathrm{T}}(x), \ldots, \mathscr{F}_{q}^{\mathrm{T}}(x)\right]^{\mathrm{T}}$, where $\mathscr{F}_{i}(x) \triangleq f_{i}\left(x_{i}\right)+\mathscr{I}_{i}(x), i=$ $1, \ldots, q, \quad G(x) \triangleq \operatorname{diag}\left[G_{1}\left(x_{1}\right), \ldots, G_{q}\left(x_{q}\right)\right], \quad h(x) \triangleq\left[h_{1}^{\mathrm{T}}\left(x_{1}\right), \ldots, h_{q}^{\mathrm{T}}\left(x_{q}\right)\right]^{\mathrm{T}}$, and $J(x) \triangleq$ $\operatorname{diag}\left[J_{1}\left(x_{1}\right), \ldots, J_{q}\left(x_{q}\right)\right]$. In addition, for all $i=1, \ldots, q$, define $\hat{R}_{i} \in \mathbb{S}^{m}, \hat{S}_{i} \in \mathbb{R}^{l \times m}$, and $\hat{Q}_{i} \in \mathbb{S}^{l}$ such that each of these matrices consists of zero blocks except, respectively, for the matrix blocks $R_{i} \in \mathbb{S}^{m_{i}}, S_{i} \in \mathbb{R}^{l_{i} \times m_{i}}$, and $Q_{i} \in \mathbb{S}^{l_{i}}$ on $(i, i)$ position. Finally, we introduce a more general definition of vector dissipativity involving an underlying nonlinear comparison system.

Definition 4.1. The discrete-time large-scale nonlinear dynamical system $\mathscr{G}$ given by (3.1), (3.2) is vector dissipative (resp., geometrically vector dissipative) with respect to the vector supply rate $S(u, y)$ if there exist a continuous, nonnegative definite vector function $V_{\mathrm{s}}=$ $\left[v_{s 1}, \ldots, v_{s q}\right]^{\mathrm{T}}: \mathscr{D} \rightarrow \overline{\mathbb{R}}_{+}^{q}$, called a vector storage function, and a class $\mathscr{W}$ function $w: \overline{\mathbb{R}}_{+}^{q} \rightarrow$ $\mathbb{R}^{q}$ such that $V_{s}(0)=0, w(0)=0$, the zero solution $r(k) \equiv 0$ to the comparison system

$$
r(k+1)=w(r(k)), \quad r\left(k_{0}\right)=r_{0}, \quad k \geq k_{0},
$$

is Lyapunov (resp., asymptotically) stable, and the vector dissipation inequality

$$
V_{s}(x(k+1)) \leq \leq w\left(V_{s}(x(k))\right)+S(u(k), y(k)), \quad k \geq k_{0},
$$

is satisfied, where $x(k), k \geq k_{0}$, is the solution to (3.1) with $u \in \mathcal{U}$. The discrete-time largescale nonlinear dynamical system $\varphi$ given by (3.1), (3.2) is vector lossless with respect to the vector supply rate $S(u, y)$ if the vector dissipation inequality is satisfied as an equality with the zero solution $r(k) \equiv 0$ to (4.1) being Lyapunov stable.

Remark 4.2. If in Definition 4.1 the function $w: \overline{\mathbb{R}}_{+}^{q} \rightarrow \mathbb{R}^{q}$ is such that $w(r)=W r$, where $W \in \mathbb{R}^{q \times q}$, then $W$ is nonnegative and Definition 4.1 collapses to Definition 3.2.

Theorem 4.3. Consider the discrete-time large-scale nonlinear dynamical system $G$ given by (3.1), (3.2). Let $R_{i} \in \mathbb{S}^{m_{i}}, S_{i} \in \mathbb{R}^{l_{i} \times m_{i}}$, and $Q_{i} \in \mathbb{S}^{l_{i}}, i=1, \ldots, q$. If there exist functions $V_{\mathrm{s}}=\left[v_{\mathrm{s} 1}, \ldots, v_{\mathrm{s} q}\right]^{\mathrm{T}}: \mathbb{R}^{n} \rightarrow \overline{\mathbb{R}}_{+}^{q}, P_{1 i}: \mathbb{R}^{n} \rightarrow \mathbb{R}^{1 \times m}, P_{2 i}: \mathbb{R}^{n} \rightarrow \mathbb{N}^{m}, w=\left[w_{1}, \ldots, w_{q}\right]^{\mathrm{T}}: \overline{\mathbb{R}}_{+}^{q} \rightarrow$ $\mathbb{R}^{q}, \ell_{i}: \mathbb{R}^{n} \rightarrow \mathbb{R}^{s_{i}}$, and $\mathscr{L}_{i}: \mathbb{R}^{n} \rightarrow \mathbb{R}^{s_{i} \times m}$, such that $v_{\mathrm{s} i}(\cdot)$ is continuous, $v_{\mathrm{s} i}(0)=0, i=1, \ldots, q$, $w \in \mathscr{W}, w(0)=0$,

$$
v_{s i}(\mathscr{F}(x)+G(x) u)=v_{s i}(\mathscr{F}(x))+P_{1 i}(x) u+u^{\mathrm{T}} P_{2 i}(x) u, \quad x \in \mathbb{R}^{n}, u \in \mathbb{R}^{m}
$$

the zero solution $r(k) \equiv 0$ to (4.1) is Lyapunov (resp., asymptotically) stable, and, for all $x \in \mathbb{R}^{n}$ and $i=1, \ldots, q$,

$$
\begin{aligned}
& 0=v_{s i}(\mathscr{F}(x))-h^{\mathrm{T}}(x) \hat{Q}_{i} h(x)-w_{i}\left(V_{\mathrm{s}}(x)\right)+\ell_{i}^{\mathrm{T}}(x) \ell_{i}(x), \\
& 0=\frac{1}{2} P_{1 i}(x)-h^{\mathrm{T}}(x)\left(\hat{S}_{i}+\hat{Q}_{i} J(x)\right)+\ell_{i}^{\mathrm{T}}(x) \mathscr{L}_{i}(x), \\
& 0=\hat{R}_{i}+J^{\mathrm{T}}(x) \hat{S}_{i}+\hat{S}_{i}^{\mathrm{T}} J(x)+J^{\mathrm{T}}(x) \hat{Q}_{i} J(x)-P_{2 i}(x)-\mathscr{L}_{i}^{\mathrm{T}}(x) \mathscr{L}_{i}(x),
\end{aligned}
$$


then $G$ is vector dissipative (resp., geometrically vector dissipative) with respect to the vector quadratic supply rate $S(u, y)$, where $s_{i}\left(u_{i}, y_{i}\right)=u_{i}^{\mathrm{T}} R_{i} u_{i}+2 y_{i}^{\mathrm{T}} S_{i} u_{i}+y_{i}^{\mathrm{T}} Q_{i} y_{i}, i=1, \ldots, q$.

Proof. Suppose that there exist functions $v_{s i}: \mathbb{R}^{n} \rightarrow \overline{\mathbb{R}}_{+}, \ell_{i}: \mathbb{R}^{n} \rightarrow \mathbb{R}^{s_{i}}, \mathscr{L}_{i}: \mathbb{R}^{n} \rightarrow \mathbb{R}^{s_{i} \times m}$, $w: \overline{\mathbb{R}}_{+}^{q} \rightarrow \mathbb{R}^{q}, P_{1 i}: \mathbb{R}^{n} \rightarrow \mathbb{R}^{1 \times m}$, and $P_{2 i}: \mathbb{R}^{n} \rightarrow \mathbb{N}^{m}$, such that $v_{s i}(\cdot)$ is continuous and nonnegative-definite, $v_{\text {si }}(0)=0, i=1, \ldots, q, w(0)=0, w \in \mathcal{W}$, the zero solution $r(k) \equiv 0$ to (4.1) is Lyapunov (resp., asymptotically) stable, and (4.3) and (4.4) are satisfied. Then for any $u \in \mathcal{U}$ and $x \in \mathbb{R}^{n}, i=1, \ldots, q$, it follows from (4.3) and (4.4) that

$$
\begin{aligned}
s_{i}\left(u_{i}, y_{i}\right)= & u^{\mathrm{T}} \hat{R}_{i} u+2 y^{\mathrm{T}} \hat{S}_{i} u+y^{\mathrm{T}} \hat{Q}_{i} y \\
= & h^{\mathrm{T}}(x) \hat{Q}_{i} h(x)+2 h^{\mathrm{T}}(x)\left(\hat{S}_{i}+\hat{Q}_{i} J(x)\right) u \\
& +u^{\mathrm{T}}\left(J^{\mathrm{T}}(x) \hat{Q}_{i} J(x)+J^{\mathrm{T}}(x) \hat{S}_{i}+\hat{S}_{i}^{\mathrm{T}} J(x)+\hat{R}_{i}\right) u \\
= & v_{\mathrm{si}}(\mathscr{F}(x))-w_{i}\left(V_{\mathrm{s}}(x)\right)+P_{1 i}(x) u+\ell_{i}^{\mathrm{T}}(x) \ell_{i}(x)+2 \ell_{i}^{\mathrm{T}}(x) \mathscr{E}_{i}(x) u \\
& +u^{\mathrm{T}} P_{2 i}(x) u+u^{\mathrm{T}} \mathscr{L}_{i}^{\mathrm{T}}(x) \mathscr{E}_{i}(x) u \\
= & v_{\mathrm{si}}(\mathscr{F}(x)+G(x) u)+\left[\ell_{i}(x)+\mathscr{L}_{i}(x) u\right]^{\mathrm{T}}\left[\ell_{i}(x)+\mathscr{L}_{i}(x) u\right]-w_{i}\left(V_{\mathrm{s}}(x)\right) \\
\geq & v_{\mathrm{si}}(\mathscr{F}(x)+G(x) u)-w_{i}\left(V_{\mathrm{s}}(x)\right),
\end{aligned}
$$

where $x(k), k \geq k_{0}$, satisfies (3.1). Now, the result follows from (4.5) with vector storage function $V_{\mathrm{s}}(x)=\left[v_{\mathrm{s} 1}(x), \ldots, v_{\mathrm{s} q}(x)\right]^{\mathrm{T}}, x \in \mathbb{R}^{n}$.

Using (4.4), it follows that for $k \geq k_{0}$ and $i=1, \ldots, q$,

$$
\begin{aligned}
& s_{i}\left(u_{i}(k), y_{i}(k)\right)+\left[w_{i}\left(V_{s}(x(k))\right)-v_{s i}(x(k))\right] \\
& \quad=\Delta v_{s i}(x(k))+\left[\ell_{i}(x(k))+\mathscr{L}_{i}(x(k)) u(k)\right]^{\mathrm{T}}\left[\ell_{i}(x(k))+\mathscr{L}_{i}(x(k)) u(k)\right],
\end{aligned}
$$

where $V_{s}(x)=\left[v_{\mathrm{s} 1}(x), \ldots, v_{\mathrm{s} q}(x)\right]^{\mathrm{T}}, x \in \mathbb{R}^{n}$, which can be interpreted as a generalized energy balance equation for the $i$ th subsystem of $G_{\text {where }} \Delta v_{s i}(x(k))$ is the change in energy between consecutive discrete times, the two discrete terms on the left are, respectively, the external supplied energy to the $i$ th subsystem and the energy gained by the $i$ th subsystem from the net energy flow between all subsystems due to subsystem coupling, and the second discrete term on the right corresponds to the dissipated energy from the $i$ th subsystem.

Remark 4.4. Note that if $\mathscr{G}$ with $u(k) \equiv 0$ is vector dissipative (resp., geometrically vector dissipative) with respect to the vector quadratic supply rate where $Q_{i} \leq 0, i=1, \ldots, q$, then it follows from the vector dissipation inequality that

$$
V_{s}(x(k+1)) \leq \leq w\left(V_{s}(x(k))\right)+S(0, y(k)) \leq \leq w\left(V_{s}(x(k))\right), \quad k \geq k_{0},
$$


where $S(0, y)=\left[s_{1}\left(0, y_{1}\right), \ldots, s_{q}\left(0, y_{q}\right)\right]^{\mathrm{T}}, s_{i}\left(0, y_{i}(k)\right)=y_{i}^{\mathrm{T}}(k) Q_{i} y_{i}(k) \leq 0, k \geq k_{0}, i=1, \ldots, q$, and $x(k), k \geq k_{0}$, is the solution to (3.1) with $u(k) \equiv 0$. If, in addition, there exists $p \in \mathbb{R}_{+}^{q}$ such that $p^{\mathrm{T}} V_{s}(x), x \in \mathbb{R}^{n}$, is positive definite, then it follows from Theorem 2.7 that the undisturbed $(u(k) \equiv 0)$ large-scale nonlinear dynamical system (3.1) is Lyapunov (resp., asymptotically) stable.

Next, we extend the notions of passivity and nonexpansivity to vector passivity and vector nonexpansivity.

Definition 4.5. The discrete-time large-scale nonlinear dynamical system $\mathscr{G}$ given by (3.1), (3.2) with $m_{i}=l_{i}, i=1, \ldots, q$, is vector passive (resp., geometrically vector passive) if it is vector dissipative (resp., geometrically vector dissipative) with respect to the vector supply rate $S(u, y)$, where $s_{i}\left(u_{i}, y_{i}\right)=2 y_{i}^{\mathrm{T}} u_{i}, i=1, \ldots, q$.

Definition 4.6. The discrete-time large-scale nonlinear dynamical system $\varphi$ given by (3.1), (3.2) is vector nonexpansive (resp., geometrically vector nonexpansive) if it is vector dissipative (resp., geometrically vector dissipative) with respect to the vector supply rate $S(u, y)$, where $s_{i}\left(u_{i}, y_{i}\right)=\gamma_{i}^{2} u_{i}^{\mathrm{T}} u_{i}-y_{i}^{\mathrm{T}} y_{i}, i=1, \ldots, q$, and $\gamma_{i}>0, i=1, \ldots, q$, are given.

Remark 4.7. Note that a mixed vector passive nonexpansive formulation of $\mathscr{G}$ can also be considered. Specifically, one can consider discrete-time large-scale nonlinear dynamical systems $\mathscr{G}_{\text {which }}$ are vector dissipative with respect to vector supply rate $S(u, y)$, where $s_{i}\left(u_{i}, y_{i}\right)=2 y_{i}^{\mathrm{T}} u_{i}, i \in \mathbb{Z}_{\mathrm{p}}, s_{j}\left(u_{j}, y_{j}\right)=\gamma_{j}^{2} u_{j}^{\mathrm{T}} u_{j}-y_{j}^{\mathrm{T}} y_{j}, \gamma_{j}>0, j \in \mathbb{Z}_{\text {ne }}$, and $\mathbb{Z}_{\mathrm{p}} \cup$ $\mathbb{Z}_{\mathrm{ne}}=\{1, \ldots, q\}$. Furthermore, vector supply rates for vector input strict passivity, vector output strict passivity, and vector input-output strict passivity, generalizing the passivity notions given in [10], can also be considered. However, for simplicity of exposition, we do not do so here.

The next result presents constructive sufficient conditions guaranteeing vector dissipativity of $G_{\text {with }}$ respect to a vector quadratic supply rate for the case where the vector storage function $V_{s}(x), x \in \mathbb{R}^{n}$, is component decoupled; that is, $V_{s}(x)=\left[v_{s 1}\left(x_{1}\right), \ldots, v_{s q}\left(x_{q}\right)\right]^{\mathrm{T}}$, $x \in \mathbb{R}^{n}$.

Theorem 4.8. Consider the discrete-time large-scale nonlinear dynamical system $\mathscr{G}$ given by (3.1), (3.2). Assume that there exist functions $V_{\mathrm{s}}=\left[v_{\mathrm{s} 1}, \ldots, v_{\mathrm{sq}}\right]^{\mathrm{T}}: \mathbb{R}^{n} \rightarrow \overline{\mathbb{R}}_{+}^{q}, P_{1 i}: \mathbb{R}^{n} \rightarrow$ $\mathbb{R}^{1 \times m_{i}}, P_{2 i}: \mathbb{R}^{n} \rightarrow \mathbb{N}^{m_{i}}, w=\left[w_{1}, \ldots, w_{q}\right]^{\mathrm{T}}: \overline{\mathbb{R}}_{+}^{q} \rightarrow \mathbb{R}^{q}, \ell_{i}: \mathbb{R}^{n} \rightarrow \mathbb{R}^{s_{i}}$, and $\mathscr{E}_{i}: \mathbb{R}^{n} \rightarrow \mathbb{R}^{s_{i} \times m_{i}}$ such that $v_{s i}(\cdot)$ is continuous, $v_{s i}(0)=0, i=1, \ldots, q, w \in \mathcal{W}, w(0)=0$, the zero solution $r(k) \equiv 0$ to (4.1) is Lyapunov (resp., asymptotically) stable, and, for all $x \in \mathbb{R}^{n}$ and $i=$ $1, \ldots, q$,

$$
\begin{aligned}
& 0 \leq v_{\mathrm{s} i}\left(\mathscr{F}_{i}(x)\right)-v_{\mathrm{si}}\left(\mathscr{F}_{i}(x)+G_{i}\left(x_{i}\right) u_{i}\right)+P_{1 i}(x) u_{i}+u_{i}^{\mathrm{T}} P_{2 i}(x) u_{i}, \\
& 0 \geq v_{\mathrm{s} i}\left(\mathscr{F}_{i}(x)\right)-h_{i}^{\mathrm{T}}\left(x_{i}\right) Q_{i} h_{i}\left(x_{i}\right)-w_{i}\left(V_{\mathrm{s}}(x)\right)+\ell_{i}^{\mathrm{T}}\left(x_{i}\right) \ell_{i}\left(x_{i}\right), \\
& 0=\frac{1}{2} P_{1 i}(x)-h_{i}^{\mathrm{T}}\left(x_{i}\right)\left(S_{i}+Q_{i} J_{i}\left(x_{i}\right)\right)+\ell_{i}^{\mathrm{T}}\left(x_{i}\right) \mathscr{L}_{i}\left(x_{i}\right), \\
& 0 \leq R_{i}+J_{i}^{\mathrm{T}}\left(x_{i}\right) S_{i}+S_{i}^{\mathrm{T}} J_{i}\left(x_{i}\right)+J_{i}^{\mathrm{T}}\left(x_{i}\right) Q_{i} J_{i}\left(x_{i}\right)-P_{2 i}(x)-\mathscr{L}_{i}^{\mathrm{T}}\left(x_{i}\right) \mathscr{L}_{i}\left(x_{i}\right) .
\end{aligned}
$$


Then $G$ is vector dissipative (resp., geometrically vector dissipative) with respect to the vector supply rate $S(u, y)$, where $s_{i}\left(u_{i}, y_{i}\right)=u_{i}^{\mathrm{T}} R_{i} u_{i}+2 y_{i}^{\mathrm{T}} S_{i} u_{i}+y_{i}^{\mathrm{T}} Q_{i} y_{i}, i=1, \ldots, q$.

Proof. For any admissible input $u=\left[u_{1}^{\mathrm{T}}, \ldots, u_{q}^{\mathrm{T}}\right]^{\mathrm{T}}$ such that $u_{i} \in \mathbb{R}^{m_{i}}, k \in \overline{\mathbb{Z}}_{+}$, and $i=$ $1, \ldots, q$, it follows from $(4.8)$ that

$$
\begin{aligned}
s_{i}\left(u_{i}(k), y_{i}(k)\right)= & u_{i}^{\mathrm{T}}(k) R_{i} u_{i}(k)+2 y_{i}^{\mathrm{T}}(k) S_{i} u_{i}(k)+y_{i}^{\mathrm{T}}(k) Q_{i} y_{i}(k) \\
= & h_{i}^{\mathrm{T}}\left(x_{i}(k)\right) Q_{i} h_{i}\left(x_{i}(k)\right)+2 h_{i}^{\mathrm{T}}\left(x_{i}(k)\right)\left(S_{i}+Q_{i} J_{i}\left(x_{i}(k)\right)\right) u_{i}(k) \\
& +u_{i}^{\mathrm{T}}(k)\left(J_{i}^{\mathrm{T}}\left(x_{i}(k)\right) Q_{i} J_{i}\left(x_{i}(k)\right)+J_{i}^{\mathrm{T}}\left(x_{i}(k)\right) S_{i}+S_{i}^{\mathrm{T}} J_{i}\left(x_{i}(k)\right)+R_{i}\right) u_{i}(k) \\
\geq & v_{s i}\left(\mathscr{F}_{i}(x(k))\right)+P_{1 i}(x(k)) u_{i}(k)+\ell_{i}^{\mathrm{T}}\left(x_{i}(k)\right) \ell_{i}\left(x_{i}(k)\right) \\
& +2 \ell_{i}^{\mathrm{T}}\left(x_{i}(k)\right) \mathscr{L}_{i}\left(x_{i}(k)\right) u_{i}(k)+u_{i}^{\mathrm{T}}(k) P_{2 i}(x(k)) u_{i}(k) \\
& +u_{i}^{\mathrm{T}}(k) \mathscr{L}_{i}^{\mathrm{T}}\left(x_{i}(k)\right) \mathscr{L}_{i}\left(x_{i}(k)\right) u_{i}(k)-w_{i}\left(V_{\mathrm{s}}(x(k))\right) \\
\geq & v_{\mathrm{si}}\left(x_{i}(k+1)\right)+\left[\ell_{i}\left(x_{i}(k)\right)+\mathscr{L}_{i}\left(x_{i}(k)\right) u_{i}(k)\right]^{\mathrm{T}} \\
& \times\left[\ell_{i}\left(x_{i}(k)\right)+\mathscr{L}_{i}\left(x_{i}(k)\right) u_{i}(k)\right]-w_{i}\left(V_{\mathrm{s}}(x(k))\right) \\
\geq & v_{s i}\left(x_{i}(k+1)\right)-w_{i}\left(V_{\mathrm{s}}(x(k))\right),
\end{aligned}
$$

where $x(k), k \geq k_{0}$, satisfies (3.1). Now, the result follows from (4.9) with vector storage function $V_{s}(x)=\left[v_{s 1}\left(x_{1}\right), \ldots, v_{s q}\left(x_{q}\right)\right]^{\mathrm{T}}, x \in \mathbb{R}^{n}$.

Finally, we provide necessary and sufficient conditions for the case where the discretetime large-scale nonlinear dynamical system $\mathscr{G}$ is vector lossless with respect to a vector quadratic supply rate.

Theorem 4.9. Consider the discrete-time large-scale nonlinear dynamical system $G$ given by (3.1), (3.2). Let $R_{i} \in \mathbb{S}^{m_{i}}, S_{i} \in \mathbb{R}^{l_{i} \times m_{i}}$, and $Q_{i} \in \mathbb{S}^{l_{i}}, i=1, \ldots, q$. Then $\varphi$ is vector lossless with respect to the vector quadratic supply rate $S(u, y)$, where $s_{i}\left(u_{i}, y_{i}\right)=u_{i}^{\mathrm{T}} R_{i} u_{i}+2 y_{i}^{\mathrm{T}} S_{i} u_{i}+$ $y_{i}^{\mathrm{T}} Q_{i} y_{i}, i=1, \ldots, q$, if and only if there exist functions $V_{\mathrm{s}}=\left[v_{\mathrm{s} 1}, \ldots, v_{\mathrm{s} q}\right]^{\mathrm{T}}: \mathbb{R}^{n} \rightarrow \overline{\mathbb{R}}_{+}^{q}, P_{1 i}$ : $\mathbb{R}^{n} \rightarrow \mathbb{R}^{1 \times m}, P_{2 i}: \mathbb{R}^{n} \rightarrow \mathbb{N}^{m}$, and $w=\left[w_{1}, \ldots, w_{q}\right]^{\mathrm{T}}: \overline{\mathbb{R}}_{+}^{q} \rightarrow \mathbb{R}^{q}$ such that $v_{s i}(\cdot)$ is continuous, $v_{s i}(0)=0, i=1, \ldots, q, w \in \mathcal{W}, w(0)=0$, the zero solution $r(k) \equiv 0$ to (4.1) is Lyapunov stable, and, for all $x \in \mathbb{R}^{n}, i=1, \ldots, q$, (4.3) holds and

$$
\begin{aligned}
& 0=v_{s i}(\mathscr{F}(x))-h^{\mathrm{T}}(x) \hat{Q}_{i} h(x)-w_{i}\left(V_{s}(x)\right), \\
& 0=\frac{1}{2} P_{1 i}(x)-h^{\mathrm{T}}(x)\left(\hat{S}_{i}+\hat{Q}_{i} J(x)\right), \\
& 0=\hat{R}_{i}+J^{\mathrm{T}}(x) \hat{S}_{i}+\hat{S}_{i}^{\mathrm{T}} J(x)+J^{\mathrm{T}}(x) \hat{Q}_{i} J(x)-P_{2 i}(x) .
\end{aligned}
$$

Proof. Sufficiency follows as in the proof of Theorem 4.3. To show necessity, suppose that $\mathscr{G}$ is lossless with respect to the vector quadratic supply rate $S(u, y)$. Then, there exist continuous functions $V_{\mathrm{s}}=\left[v_{\mathrm{s} 1}, \ldots, v_{\mathrm{s} q}\right]^{\mathrm{T}}: \mathbb{R}^{n} \rightarrow \overline{\mathbb{R}}_{+}^{q}$ and $w=\left[w_{1}, \ldots, w_{q}\right]^{\mathrm{T}}: \overline{\mathbb{R}}_{+}^{q} \rightarrow \mathbb{R}^{q}$ such 
that $V_{\mathrm{s}}(0)=0$, the zero solution $r(k) \equiv 0$ to (4.1) is Lyapunov stable, and

$$
\begin{aligned}
v_{\mathrm{s} i}(\mathscr{F}(x)+G(x) u)= & w_{i}\left(V_{\mathrm{s}}(x)\right)+s_{i}\left(u_{i}, y_{i}\right) \\
= & w_{i}\left(V_{\mathrm{s}}(x)\right)+u^{\mathrm{T}} \hat{R}_{i} u+2 y^{\mathrm{T}} \hat{S}_{i} u+y^{\mathrm{T}} \hat{Q}_{i} y \\
= & w_{i}\left(V_{\mathrm{s}}(x)\right)+h^{\mathrm{T}}(x) \hat{Q}_{i} h(x)+2 h^{\mathrm{T}}(x)\left(\hat{Q}_{i} J(x)+\hat{S}_{i}\right) u \\
& +u^{\mathrm{T}}\left(\hat{R}_{i}+\hat{S}_{i}^{\mathrm{T}} J(x)+J^{\mathrm{T}}(x) \hat{S}_{i}+J^{\mathrm{T}}(x) \hat{Q}_{i} J(x)\right) u, \quad x \in \mathbb{R}^{n}, u \in \mathbb{R}^{m} .
\end{aligned}
$$

Since the right-hand side of (4.11) is quadratic in $u$, it follows that $v_{s i}(\mathscr{F}(x)+G(x) u)$ is quadratic in $u$ and hence there exist $P_{1 i}: \mathbb{R}^{n} \rightarrow \mathbb{R}^{1 \times m}$ and $P_{2 i}: \mathbb{R}^{n} \rightarrow \mathbb{N}^{m}$ such that

$$
v_{s i}(\mathscr{F}(x)+G(x) u)=v_{s i}(\mathscr{F}(x))+P_{1 i}(x) u+u^{\mathrm{T}} P_{2 i}(x) u, \quad x \in \mathbb{R}^{n}, u \in \mathbb{R}^{m} .
$$

Now, using (4.12) and equating coefficients of equal powers in (4.11) yield (4.10).

\section{Specialization to discrete-time large-scale linear dynamical systems}

In this section, we specialize the results of Section 4 to the case of discrete-time large-scale linear dynamical systems. Specifically, we assume that $w \in \mathscr{W}$ is linear so that $w(r)=$ $W r$, where $W \in \mathbb{R}^{q \times q}$ is nonnegative, and consider the discrete-time large-scale linear dynamical system $\mathscr{G}$ given by

$$
\begin{gathered}
x(k+1)=A x(k)+B u(k), \quad x\left(k_{0}\right)=x_{0}, \quad k \geq k_{0}, \\
y(k)=C x(k)+D u(k),
\end{gathered}
$$

where $A \in \mathbb{R}^{n \times n}$ and $A$ is partitioned as $A \triangleq\left[A_{i j}\right], i, j=1, \ldots, q, A_{i j} \in \mathbb{R}^{n_{i} \times n_{j}}, \sum_{i=1}^{q} n_{i}=$ $n, B=\operatorname{block}-\operatorname{diag}\left[B_{1}, \ldots, B_{q}\right], C=\operatorname{block}-\operatorname{diag}\left[C_{1}, \ldots, C_{q}\right], D=\operatorname{block}-\operatorname{diag}\left[D_{1}, \ldots, D_{q}\right]$, $B_{i} \in \mathbb{R}^{n_{i} \times m_{i}}, C_{i} \in \mathbb{R}^{l_{i} \times n_{i}}$, and $D_{i} \in \mathbb{R}^{l_{i} \times m_{i}}, i=1, \ldots, q$.

Theorem 5.1. Consider the discrete-time large-scale linear dynamical system $G$ given by

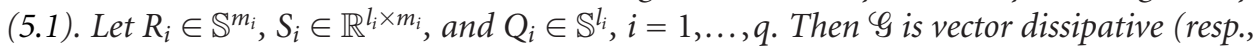
geometrically vector dissipative) with respect to the vector supply rate $S(u, y)$, where $s_{i}\left(u_{i}, y_{i}\right)$ $=u_{i}^{\mathrm{T}} R_{i} u_{i}+2 y_{i}^{\mathrm{T}} S_{i} u_{i}+y_{i}^{\mathrm{T}} Q_{i} y_{i}, i=1, \ldots, q$, and with a three-times continuously differentiable vector storage function if and only if there exist $W \in \mathbb{R}^{q \times q}, P_{i} \in \mathbb{N}^{n}, L_{i} \in \mathbb{R}^{s_{i} \times n}$, and $Z_{i} \in$ $\mathbb{R}^{s_{i} \times m}, i=1, \ldots, q$, such that $W$ is nonnegative and semistable (resp., asymptotically stable), and, for all $i=1, \ldots, q$,

$$
\begin{aligned}
& 0=A^{\mathrm{T}} P_{i} A-C^{\mathrm{T}} \hat{Q}_{i} C-\sum_{j=1}^{q} W_{(i, j)} P_{j}+L_{i}^{\mathrm{T}} L_{i}, \\
& 0=A^{\mathrm{T}} P_{i} B-C^{\mathrm{T}}\left(\hat{S}_{i}+\hat{Q}_{i} D\right)+L_{i}^{\mathrm{T}} Z_{i}, \\
& 0=\hat{R}_{i}+D^{\mathrm{T}} \hat{S}_{i}+\hat{S}_{i}^{\mathrm{T}} D+D^{\mathrm{T}} \hat{Q}_{i} D-B^{\mathrm{T}} P_{i} B-Z_{i}^{\mathrm{T}} Z_{i} .
\end{aligned}
$$


Proof. Sufficiency follows from Theorem 4.3 with $\mathscr{F}(x)=A x, G(x)=B, h(x)=C x, J(x)=$ $D, P_{1 i}(x)=2 x^{\mathrm{T}} A^{\mathrm{T}} P_{i} B, P_{2 i}(x)=B^{\mathrm{T}} P_{i} B, w(r)=W r, \ell_{i}(x)=L_{i} x, \mathscr{L}_{i}(x)=Z_{i}$, and $v_{s i}(x)=$ $x^{\mathrm{T}} P_{i} x, i=1, \ldots, q$. To show necessity, suppose $\mathscr{G}_{\text {is }}$ vector dissipative with respect to the vector supply rate $S(u, y)$, where $s_{i}\left(u_{i}, y_{i}\right)=u_{i}^{\mathrm{T}} R_{i} u_{i}+2 y_{i}^{\mathrm{T}} S_{i} u_{i}+y_{i}^{\mathrm{T}} Q_{i} y_{i}, i=1, \ldots, q$. Then, with $w(r)=W r$, there exists $V_{s}: \mathbb{R}^{n} \rightarrow \overline{\mathbb{R}}_{+}^{q}$ such that $W$ is nonnegative and semistable (resp., asymptotically stable), $V_{\mathrm{s}}(x) \triangleq\left[v_{\mathrm{s} 1}(x), \ldots, v_{\mathrm{s} q}(x)\right]^{\mathrm{T}}, x \in \mathbb{R}^{n}, V_{\mathrm{s}}(0)=0$, and for all $x \in \mathbb{R}^{n}, u \in \mathbb{R}^{n}$,

$$
V_{\mathrm{s}}(A x+B u)-W V_{\mathrm{s}}(x) \leq \leq S(u, y) .
$$

Next, it follows from (5.3) that there exists a three-times continuously differentiable vector function $d=\left[d_{1}, \ldots, d_{q}\right]^{\mathrm{T}}: \mathbb{R}^{n} \times \mathbb{R}^{m} \rightarrow \mathbb{R}^{q}$ such that $d(x, u) \geq \geq 0, d(0,0)=0$, and

$$
0=V_{\mathrm{s}}(A x+B u)-W V_{s}(x)-S(u, C x+D u)+d(x, u) .
$$

Now, expanding $v_{s i}(\cdot)$ and $d_{i}(\cdot, \cdot)$ via Taylor series expansion about $x=0, u=0$, and using the fact that $v_{s i}(\cdot)$ and $d_{i}(\cdot, \cdot)$ are nonnegative and $v_{s i}(0)=0, d_{i}(0,0)=0, i=1, \ldots, q$, it follows that there exist $P_{i} \in \mathbb{N}^{n}, L_{i} \in \mathbb{R}^{s_{i} \times n}$, and $Z_{i} \in \mathbb{R}^{s_{i} \times m}, i=1, \ldots, q$, such that

$$
\begin{gathered}
v_{s i}(x)=x^{\mathrm{T}} P_{i} x+v_{\text {sri }}(x), \\
d_{i}(x, u)=\left(L_{i} x+Z_{i} u\right)^{\mathrm{T}}\left(L_{i} x+Z_{i} u\right)+d_{\mathrm{ri}}(x, u), \quad x \in \mathbb{R}^{n}, u \in \mathbb{R}^{m}, i=1, \ldots, q,
\end{gathered}
$$

where $v_{\text {sri }}: \mathbb{R}^{n} \rightarrow \mathbb{R}$ and $d_{\mathrm{r} i}: \mathbb{R}^{n} \times \mathbb{R}^{m} \rightarrow \mathbb{R}$ contain the higher-order terms of $v_{\mathrm{si}}(\cdot), d_{i}(\cdot, \cdot)$, respectively. Using the above expressions, (5.4) can be written componentwise as

$$
\begin{aligned}
0= & (A x+B u)^{\mathrm{T}} P_{i}(A x+B u)-\sum_{j=1}^{q} W_{(i, j)} x^{\mathrm{T}} P_{j} x \\
& -\left(x^{\mathrm{T}} C^{\mathrm{T}} \hat{Q}_{i} C x+2 x^{\mathrm{T}} C^{\mathrm{T}} \hat{Q}_{i} D u+u^{\mathrm{T}} D^{\mathrm{T}} \hat{Q}_{i} D u+2 x^{\mathrm{T}} C^{\mathrm{T}} \hat{S}_{i} u+2 u^{\mathrm{T}} D^{\mathrm{T}} \hat{S}_{i} u+u^{\mathrm{T}} \hat{R}_{i} u\right) \\
& +\left(L_{i} x+Z_{i} u\right)^{\mathrm{T}}\left(L_{i} x+Z_{i} u\right)+\delta(x, u),
\end{aligned}
$$

where $\delta(x, u)$ is such that

$$
\lim _{\|x\|^{2}+\|u\|^{2} \rightarrow 0} \frac{|\delta(x, u)|}{\|x\|^{2}+\|u\|^{2}}=0
$$

Now, viewing (5.6) as the componentwise Taylor series expansion of (5.4) about $x=0$ and $u=0$, it follows that for all $x \in \mathbb{R}^{n}$ and $u \in \mathbb{R}^{m}$,

$$
\begin{aligned}
0= & x^{\mathrm{T}}\left(A^{\mathrm{T}} P_{i} A-\sum_{j=1}^{q} W_{(i, j)} P_{j}-C^{\mathrm{T}} \hat{Q}_{i} C+L_{i}^{\mathrm{T}} L_{i}\right) x \\
& +2 x^{\mathrm{T}}\left(A^{\mathrm{T}} P_{i} B-C^{\mathrm{T}} \hat{S}_{i}-C^{\mathrm{T}} \hat{Q}_{i} D+L_{i}^{\mathrm{T}} Z_{i}\right) u \\
& +u^{\mathrm{T}}\left(Z_{i}^{\mathrm{T}} Z_{i}-D^{\mathrm{T}} \hat{Q}_{i} D-D^{\mathrm{T}} \hat{S}_{i}-\hat{S}_{i}^{\mathrm{T}} D-\hat{R}_{i}+B^{\mathrm{T}} P_{i} B\right) u, \quad i=1, \ldots, q .
\end{aligned}
$$

Now, equating coefficients of equal powers in (5.8) yields (5.2). 
Remark 5.2. Note that the equations in (5.2) are equivalent to

$$
\left[\begin{array}{ll}
\mathscr{A}_{i} & \mathscr{B}_{i} \\
\mathscr{B}_{i}^{\mathrm{T}} & \mathscr{C}_{i}
\end{array}\right]=-\left[\begin{array}{c}
L_{i}^{\mathrm{T}} \\
Z_{i}^{\mathrm{T}}
\end{array}\right]\left[\begin{array}{ll}
L_{i} & Z_{i}
\end{array}\right] \leq 0, \quad i=1, \ldots, q,
$$

where, for all $i=1, \ldots, q$,

$$
\begin{aligned}
& \mathscr{A}_{i}=A^{\mathrm{T}} P_{i} A-C^{\mathrm{T}} \hat{Q}_{i} C-\sum_{j=1}^{q} W_{(i, j)} P_{j}, \\
& \mathscr{B}_{i}=A^{\mathrm{T}} P_{i} B-C^{\mathrm{T}}\left(\hat{S}_{i}+\hat{Q}_{i} D\right), \\
& \mathscr{C}_{i}=-\left(\hat{R}_{i}+D^{\mathrm{T}} \hat{S}_{i}+\hat{S}_{i}^{\mathrm{T}} D+D^{\mathrm{T}} \hat{Q}_{i} D-B^{\mathrm{T}} P_{i} B\right) .
\end{aligned}
$$

Hence, vector dissipativity of discrete-time large-scale linear dynamical systems with respect to vector quadratic supply rates can be characterized via (cascade) linear matrix inequalities (LMIs) [5]. A similar remark holds for Theorem 5.3 below.

The next result presents sufficient conditions guaranteeing vector dissipativity of $\mathscr{G}$ with respect to a vector quadratic supply rate in the case where the vector storage function is component decoupled.

Theorem 5.3. Consider the discrete-time large-scale linear dynamical system $G$ given by (5.1). Let $R_{i} \in \mathbb{S}^{m_{i}}, S_{i} \in \mathbb{R}^{l_{i} \times m_{i}}$, and $Q_{i} \in \mathbb{S}^{l_{i}}, i=1, \ldots, q$, be given. Assume there exist matrices $W \in \mathbb{R}^{q \times q}, P_{i} \in \mathbb{N}^{n_{i}}, L_{i i} \in \mathbb{R}^{s_{i i} \times n_{i}}, Z_{i i} \in \mathbb{R}^{s_{i i} \times m_{i}}, i=1, \ldots, q, L_{i j} \in \mathbb{R}^{s_{i j} \times n_{i}}$, and $Z_{i j} \in$ $\mathbb{R}^{s_{i j} \times n_{j}}, i, j=1, \ldots, q, i \neq j$, such that $W$ is nonnegative and semistable (resp., asymptotically stable), and, for all $i=1, \ldots, q$,

$$
\begin{aligned}
& 0 \geq A_{i i}^{\mathrm{T}} P_{i} A_{i i}-C_{i}^{\mathrm{T}} Q_{i} C_{i}-W_{(i, i)} P_{i}+L_{i i}^{\mathrm{T}} L_{i i}+\sum_{j=1, j \neq i}^{q} L_{i j}^{\mathrm{T}} L_{i j}, \\
& 0=A_{i i}^{\mathrm{T}} P_{i} B_{i}-C_{i}^{\mathrm{T}} S_{i}-C_{i}^{\mathrm{T}} Q_{i} D_{i}+L_{i i}^{\mathrm{T}} Z_{i i}, \\
& 0 \leq R_{i}+D_{i}^{\mathrm{T}} S_{i}+S_{i}^{\mathrm{T}} D_{i}+D_{i}^{\mathrm{T}} Q_{i} D_{i}-B_{i}^{\mathrm{T}} P_{i} B_{i}-Z_{i i}^{\mathrm{T}} Z_{i i},
\end{aligned}
$$

and for $j=1, \ldots, q, l=1, \ldots, q, j \neq i, l \neq i, l \neq j$,

$$
\begin{aligned}
& 0=A_{i j}^{\mathrm{T}} P_{i} B_{i}, \\
& 0=A_{i j}^{\mathrm{T}} P_{i} A_{i l}, \\
& 0=A_{i i}^{\mathrm{T}} P_{i} A_{i j}+L_{i j}^{\mathrm{T}} Z_{i j}, \\
& 0 \leq W_{(i, j)} P_{j}-Z_{i j}^{\mathrm{T}} Z_{i j}-A_{i j}^{\mathrm{T}} P_{i} A_{i j} .
\end{aligned}
$$

Then $G$ is vector dissipative (resp., geometrically vector dissipative) with respect to the vector supply rate $S(u, y) \triangleq\left[s_{1}\left(u_{1}, y_{1}\right), \ldots, s_{q}\left(u_{q}, y_{q}\right)\right]^{\mathrm{T}}$, where $s_{i}\left(u_{i}, y_{i}\right)=u_{i}^{\mathrm{T}} R_{i} u_{i}+2 y_{i}^{\mathrm{T}} S_{i} u_{i}+$ $y_{i}^{\mathrm{T}} Q_{i} y_{i}, i=1, \ldots, q$.

Proof. Since $P_{i} \in \mathbb{N}^{n_{i}}$, the function $v_{s i}\left(x_{i}\right) \triangleq x_{i}^{\mathrm{T}} P_{i} x_{i}, x_{i} \in \mathbb{R}^{n_{i}}$, is nonnegative definite and $v_{s i}(0)=0$. Moreover, since $v_{s i}(\cdot)$ is continuous, it follows from (5.11) and (5.12) that for 
all $u_{i} \in \mathbb{R}^{m_{i}}, i=1, \ldots, q$, and $k \geq k_{0}$,

$$
\begin{aligned}
v_{s i}\left(x_{i}(k+1)\right)= & {\left[\sum_{j=1}^{q} A_{i j} x_{j}(k)+B_{i} u_{i}(k)\right]^{\mathrm{T}} P_{i}\left[\sum_{j=1}^{q} A_{i j} x_{j}(k)+B_{i} u_{i}(k)\right] } \\
\leq & x_{i}^{\mathrm{T}}(k)\left[W_{(i, i)} P_{i}+C_{i}^{\mathrm{T}} Q_{i} C_{i}-L_{i i}^{\mathrm{T}} L_{i i}-\sum_{j=1, j \neq i}^{q} L_{i j}^{\mathrm{T}} L_{i j}\right] x_{i}(k) \\
& -\sum_{j=1, j \neq i}^{q} 2 x_{i}^{\mathrm{T}}(k) L_{i j}^{\mathrm{T}} Z_{i j} x_{j}(k)+2 x_{i}^{\mathrm{T}}(k) C_{i}^{\mathrm{T}} S_{i} u_{i}(k)+2 x_{i}^{\mathrm{T}}(k) C_{i}^{\mathrm{T}} Q_{i} D_{i} u_{i}(k) \\
& -2 x_{i}^{\mathrm{T}}(k) L_{i i}^{\mathrm{T}} Z_{i i} u_{i}(k)+\sum_{j=1, j \neq i}^{q} x_{j}^{\mathrm{T}}(k)\left[W_{(i, j)} P_{j}-Z_{i j}^{\mathrm{T}} Z_{i j}\right] x_{j}(k) \\
& +u_{i}^{\mathrm{T}}(k) R_{i} u_{i}(k)+2 u_{i}^{\mathrm{T}}(k) D_{i}^{\mathrm{T}} S_{i} u_{i}(k) \\
& +u_{i}^{\mathrm{T}}(k) D_{i}^{\mathrm{T}} Q_{i} D_{i} u_{i}(k)-u_{i}^{\mathrm{T}}(k) Z_{i i}^{\mathrm{T}} Z_{i i} u_{i}(k) \\
= & \sum_{j=1}^{q} W_{(i, j)} v_{\mathrm{s} j}\left(x_{j}(k)\right)+u_{i}^{\mathrm{T}}(k) R_{i} u_{i}(k)+2 y_{i}^{\mathrm{T}}(k) S_{i} u_{i}(k)+y_{i}^{\mathrm{T}}(k) Q_{i} y_{i}(k) \\
& -\left[L_{i i} x_{i}(k)+Z_{i i} u_{i}(k)\right]^{\mathrm{T}}\left[L_{i i} x_{i}(k)+Z_{i i} u_{i}(k)\right] \\
& -\sum_{j=1, j \neq i}^{q}\left(L_{i j} x_{i}(k)+Z_{i j} x_{j}(k)\right)^{\mathrm{T}}\left(L_{i j} x_{i}(k)+Z_{i j} x_{j}(k)\right) \\
\leq & s_{i}\left(u_{i}(k), y_{i}(k)\right)+\sum_{j=1}^{q} W_{(i, j)} v_{\mathrm{s} j}\left(x_{j}(k)\right),
\end{aligned}
$$

or, equivalently, in vector form,

$$
V_{\mathrm{s}}(x(k+1)) \leq \leq W V_{\mathrm{s}}(x(k))+S(u, y), \quad u \in \mathcal{U}, k \geq k_{0},
$$

where $V_{\mathrm{s}}(x) \triangleq\left[v_{\mathrm{s} 1}\left(x_{1}\right), \ldots, v_{\mathrm{s} q}\left(x_{q}\right)\right]^{\mathrm{T}}, x \in \mathbb{R}^{n}$. Now, it follows from Proposition 3.14 that $\mathscr{G}$ is vector dissipative (resp., geometrically vector dissipative) with respect to the vector supply rate $S(u, y)$ and with vector storage function $V_{s}(x), x \in \mathbb{R}^{n}$.

\section{Stability of feedback interconnections of discrete-time large-scale nonlinear dynamical systems}

In this section, we consider stability of feedback interconnections of discrete-time largescale nonlinear dynamical systems. Specifically, for the discrete-time large-scale dynamical system $\mathscr{G}$ given by (3.1), (3.2), we consider either a dynamic or static discrete-time large-scale feedback system $\mathscr{G}_{\mathrm{c}}$. Then, by appropriately combining vector storage functions for each system, we show stability of the feedback interconnection. We begin by considering the discrete-time large-scale nonlinear dynamical system (3.1), (3.2) with 


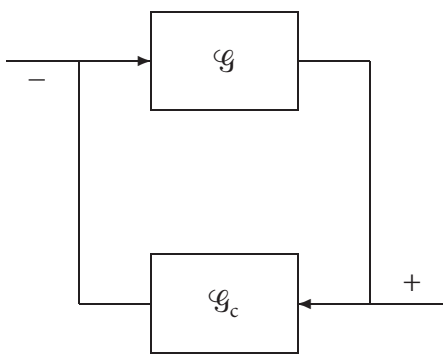

Figure 6.1. Feedback interconnection of large-scale systems $\varphi_{\text {and }} \mathscr{G}_{\mathrm{c}}$.

the large-scale feedback system $\mathscr{G}_{\mathrm{c}}$ given by

$$
\begin{gathered}
x_{\mathrm{c}}(k+1)=F_{\mathrm{c}}\left(x_{\mathrm{c}}(k), u_{\mathrm{c}}(k)\right), \quad x_{\mathrm{c}}\left(k_{0}\right)=x_{\mathrm{c} 0}, \quad k \geq k_{0}, \\
y_{\mathrm{c}}(k)=H_{\mathrm{c}}\left(x_{\mathrm{c}}(k), u_{\mathrm{c}}(k)\right),
\end{gathered}
$$

where $F_{\mathrm{c}}: \mathbb{R}^{n_{\mathrm{c}}} \times \boldsymbol{U}_{\mathrm{c}} \rightarrow \mathbb{R}^{n_{\mathrm{c}}}, H_{\mathrm{c}}: \mathbb{R}^{n_{\mathrm{c}}} \times \boldsymbol{U}_{\mathrm{c}} \rightarrow 9_{\mathrm{c}}, F_{\mathrm{c}} \triangleq\left[F_{\mathrm{c} 1}^{\mathrm{T}}, \ldots, F_{\mathrm{c} q}^{\mathrm{T}}\right]^{\mathrm{T}}, H_{\mathrm{c}} \triangleq\left[H_{\mathrm{c} 1}^{\mathrm{T}}, \ldots, H_{\mathrm{c} q}^{\mathrm{T}}\right]^{\mathrm{T}}$, $\boldsymbol{U}_{\mathrm{c}} \subseteq \mathbb{R}^{l}$, and $\mathscr{Y}_{\mathrm{c}} \subseteq \mathbb{R}^{m}$. Moreover, for all $i=1, \ldots, q$, we assume that

$$
\begin{aligned}
F_{\mathrm{c} i}\left(x_{\mathrm{c}}, u_{\mathrm{c} i}\right) & =f_{\mathrm{c} i}\left(x_{\mathrm{c} i}\right)+\Phi_{\mathrm{c} i}\left(x_{\mathrm{c}}\right)+G_{\mathrm{c} i}\left(x_{\mathrm{c} i}\right) u_{\mathrm{c} i}, \\
H_{\mathrm{c} i}\left(x_{\mathrm{c} i}, u_{\mathrm{c} i}\right) & =h_{\mathrm{c} i}\left(x_{\mathrm{c} i}\right)+J_{\mathrm{c} i}\left(x_{\mathrm{c} i}\right) u_{\mathrm{c} i},
\end{aligned}
$$

where $u_{\mathrm{c} i} \in \mathcal{U}_{\mathrm{c} i} \subseteq \mathbb{R}^{l_{i}}, y_{\mathrm{c} i} \triangleq H_{\mathrm{c} i}\left(x_{\mathrm{c} i}, u_{\mathrm{c} i}\right) \in \mathscr{Y}_{i} \subseteq \mathbb{R}^{m_{i}},\left(u_{\mathrm{c} i}, y_{\mathrm{c} i}\right)$ is the input-output pair for the $i$ th subsystem of $\varphi_{c}, f_{c i}: \mathbb{R}^{n_{c i}} \rightarrow \mathbb{R}^{n_{c i}}$ and $\Phi_{c i}: \mathbb{R}^{n_{c}} \rightarrow \mathbb{R}^{n_{c i}}$ satisfy $f_{c i}(0)=0$ and $\mathscr{I}_{c i}(0)=$ $0, G_{\mathrm{c} i}: \mathbb{R}^{n_{\mathrm{c} i}} \rightarrow \mathbb{R}^{n_{\mathrm{c} i} \times l_{i}}, h_{\mathrm{c} i}: \mathbb{R}^{n_{\mathrm{c} i}} \rightarrow \mathbb{R}^{m_{i}}$ satisfies $h_{\mathrm{c} i}(0)=0, J_{\mathrm{c} i}: \mathbb{R}^{n_{\mathrm{c} i}} \rightarrow \mathbb{R}^{m_{i} \times l_{i}}$, and $\sum_{i=1}^{q} n_{\mathrm{c} i}=$ $n_{\mathrm{c}}$. Furthermore, we define the composite input and composite output for the system $\mathscr{G}_{\mathrm{c}}$ as $u_{\mathrm{c}} \triangleq\left[u_{\mathrm{c} 1}^{\mathrm{T}}, \ldots, u_{\mathrm{c} q}^{\mathrm{T}}\right]^{\mathrm{T}}$ and $y_{\mathrm{c}} \triangleq\left[y_{\mathrm{c} 1}^{\mathrm{T}}, \ldots, y_{\mathrm{c} q}^{\mathrm{T}}\right]^{\mathrm{T}}$, respectively. In this case, $\boldsymbol{u}_{\mathrm{c}}=\boldsymbol{u}_{\mathrm{c} 1} \times \cdots \times$ $u_{c q}$ and $\mathscr{y}_{c}=\mathscr{Y}_{c 1} \times \cdots \times \mathscr{Y}_{c q}$. Note that, with the feedback interconnection given in Figure 6.1, $u_{\mathrm{c}}=y$ and $y_{\mathrm{c}}=-u$. We assume that the negative feedback interconnection of $\mathscr{G}_{\mathrm{S}}$ and $\mathscr{G}_{\mathrm{c}}$ is well posed; that is, $\operatorname{det}\left(I_{m_{i}}+J_{\mathrm{c} i}\left(x_{\mathrm{c} i}\right) J_{i}\left(x_{i}\right)\right) \neq 0$ for all $x_{i} \in \mathbb{R}^{n_{i}}$, and $x_{\mathrm{c} i} \in \mathbb{R}^{n_{\mathrm{c} i}}$, $i=1, \ldots, q$. Furthermore, we assume that for the discrete-time large-scale systems $\mathscr{G}_{\mathrm{S}}$ and $\mathscr{G}_{\mathrm{c}}$, the conditions of Theorem 3.8 are satisfied; that is, if $V_{\mathrm{s}}(x), x \in \mathbb{R}^{n}$, and $V_{\mathrm{cs}}\left(x_{\mathrm{c}}\right), x_{\mathrm{c}} \in$ $\mathbb{R}^{n_{\mathrm{c}}}$, are vector storage functions for $\varphi_{\mathcal{G}}$ and $\mathscr{G}_{\mathrm{c}}$, respectively, then there exist $p \in \mathbb{R}_{+}^{q}$ and $p_{\mathrm{c}} \in \mathbb{R}_{+}^{q}$ such that the functions $v_{\mathrm{s}}(x)=p^{\mathrm{T}} V_{\mathrm{s}}(x), x \in \mathbb{R}^{n}$, and $v_{\mathrm{cs}}\left(x_{\mathrm{c}}\right)=p_{\mathrm{c}}^{\mathrm{T}} V_{\mathrm{cs}}\left(x_{\mathrm{c}}\right), x_{\mathrm{c}} \in$ $\mathbb{R}^{n_{c}}$, are positive definite. The following result gives sufficient conditions for Lyapunov and asymptotic stability of the feedback interconnection given in Figure 6.1.

Theorem 6.1. Consider the discrete-time large-scale nonlinear dynamical systems $\mathscr{G}_{\text {and }} \mathscr{G}_{\mathrm{c}}$ given by (3.1), (3.2), and (6.1), respectively. Assume that $G_{G}$ and $G_{\mathrm{c}}$ are vector dissipative with respect to the vector supply rates $S(u, y)$ and $S_{c}\left(u_{c}, y_{c}\right)$, and with continuous vector storage functions $V_{\mathrm{s}}(\cdot)$ and $V_{\mathrm{cs}}(\cdot)$ and dissipation matrices $W \in \mathbb{R}^{q \times q}$ and $W_{\mathrm{c}} \in \mathbb{R}^{q \times q}$, respectively. 
(i) If there exists $\Sigma \triangleq \operatorname{diag}\left[\sigma_{1}, \ldots, \sigma_{q}\right]>0$ such that $S(u, y)+\Sigma S_{\mathrm{c}}\left(u_{\mathrm{c}}, y_{\mathrm{c}}\right) \leq \leq 0$ and $\tilde{W} \in$ $\mathbb{R}^{q \times q}$ is semistable (resp., asymptotically stable), where $\tilde{W}_{(i, j)} \triangleq \max \left\{W_{(i, j)},\left(\Sigma W_{c} \Sigma^{-1}\right)_{(i, j)}\right\}$ $=\max \left\{W_{(i, j)},\left(\sigma_{i} / \sigma_{j}\right) W_{\mathrm{c}(i, j)}\right\}, i, j=1, \ldots, q$, then the negative feedback interconnection of $\mathcal{G}$ and $G_{\mathrm{c}}$ is Lyapunov (resp., asymptotically) stable.

(ii) Let $Q_{i} \in \mathbb{S}^{l_{i}}, S_{i} \in \mathbb{R}^{l_{i} \times m_{i}}, R_{i} \in \mathbb{S}^{m_{i}}, Q_{\mathrm{c} i} \in \mathbb{S}^{m_{i}}, S_{\mathrm{c} i} \in \mathbb{R}^{m_{i} \times l_{i}}$, and $R_{\mathrm{c} i} \in \mathbb{S}^{l_{i}}$, and suppose $S(u, y)=\left[s_{1}\left(u_{1}, y_{1}\right), \ldots, s_{q}\left(u_{q}, y_{q}\right)\right]^{\mathrm{T}}$ and $S_{\mathrm{c}}\left(u_{\mathrm{c}}, y_{\mathrm{c}}\right)=\left[s_{\mathrm{c} 1}\left(u_{\mathrm{c} 1}, y_{\mathrm{c} 1}\right), \ldots, s_{q}\left(u_{\mathrm{c} q}, y_{\mathrm{c} q}\right)\right]^{\mathrm{T}}$, where $s_{i}\left(u_{i}, y_{i}\right)=u_{i}^{\mathrm{T}} R_{i} u_{i}+2 y_{i}^{\mathrm{T}} S_{i} u_{i}+y_{i}^{\mathrm{T}} Q_{i} y_{i}$ and $s_{\mathrm{c} i}\left(u_{\mathrm{c} i}, y_{\mathrm{c} i}\right)=u_{\mathrm{c} i}^{\mathrm{T}} R_{\mathrm{c} i} u_{\mathrm{c} i}+2 y_{\mathrm{c} i}^{\mathrm{T}} S_{\mathrm{c} i} u_{\mathrm{c} i}+y_{\mathrm{c} i}^{\mathrm{T}} Q_{\mathrm{c} i} y_{\mathrm{c} i}, i=$ $1, \ldots, q$. If there exists $\Sigma \triangleq \operatorname{diag}\left[\sigma_{1}, \ldots, \sigma_{q}\right]>0$ such that for all $i=1, \ldots, q$,

$$
\tilde{Q}_{i} \triangleq\left[\begin{array}{cc}
Q_{i}+\sigma_{i} R_{\mathrm{c} i} & -S_{i}+\sigma_{i} S_{\mathrm{c} i}^{\mathrm{T}} \\
-S_{i}^{\mathrm{T}}+\sigma_{i} S_{\mathrm{c} i} & R_{i}+\sigma_{i} Q_{\mathrm{c} i}
\end{array}\right] \leq 0
$$

and $\tilde{W} \in \mathbb{R}^{q \times q}$ is semistable (resp., asymptotically stable), where $\tilde{W}_{(i, j)} \triangleq \max \left\{W_{(i, j)}\right.$, $\left.\left(\Sigma W_{\mathrm{c}} \Sigma^{-1}\right)_{(i, j)}\right\}=\max \left\{W_{(i, j)},\left(\sigma_{i} / \sigma_{j}\right) W_{\mathrm{c}(i, j)}\right\}, i, j=1, \ldots, q$, then the negative feedback interconnection of $\mathscr{G}_{\text {and }} \mathscr{G}_{\mathrm{c}}$ is Lyapunov (resp., asymptotically) stable.

Proof. (i) Consider the vector Lyapunov function candidate $V\left(x, x_{\mathrm{c}}\right)=V_{\mathrm{s}}(x)+\Sigma V_{\mathrm{cs}}\left(x_{\mathrm{c}}\right)$, $\left(x, x_{\mathrm{c}}\right) \in \mathbb{R}^{n} \times \mathbb{R}^{n_{\mathrm{c}}}$, and note that

$$
\begin{aligned}
V\left(x(k+1), x_{\mathrm{c}}(k+1)\right)= & V_{\mathrm{s}}(x(k+1))+\sum V_{\mathrm{cs}}\left(x_{\mathrm{c}}(k+1)\right) \\
\leq & \leq S(u(k), y(k))+\Sigma S_{\mathrm{c}}\left(u_{\mathrm{c}}(k), y_{\mathrm{c}}(k)\right) \\
& +W V_{\mathrm{s}}(x(k))+\sum W_{\mathrm{c}} V_{\mathrm{cs}}\left(x_{\mathrm{c}}(k)\right) \\
\leq & \leq W V_{\mathrm{s}}(x(k))+\sum W_{\mathrm{c}} \Sigma^{-1} \sum V_{\mathrm{cs}}\left(x_{\mathrm{c}}(k)\right) \\
\leq & \leq \tilde{W}\left(V_{\mathrm{s}}(x(k))+\sum V_{\mathrm{cs}}\left(x_{\mathrm{c}}(k)\right)\right) \\
= & \tilde{W} V\left(x(k), x_{\mathrm{c}}(k)\right), \quad\left(x(k), x_{\mathrm{c}}(k)\right) \in \mathbb{R}^{n} \times \mathbb{R}^{n_{\mathrm{c}}}, k \geq k_{0} .
\end{aligned}
$$

Next, since for $V_{s}(x), x \in \mathbb{R}^{n}$, and $V_{\mathrm{cs}}\left(x_{\mathrm{c}}\right), x_{\mathrm{c}} \in \mathbb{R}^{n_{\mathrm{c}}}$, there exist, by assumption, $p \in \mathbb{R}_{+}^{q}$ and $p_{\mathrm{c}} \in \mathbb{R}_{+}^{q}$ such that the functions $v_{\mathrm{s}}(x)=p^{\mathrm{T}} V_{\mathrm{s}}(x), x \in \mathbb{R}^{n}$, and $v_{\mathrm{cs}}\left(x_{\mathrm{c}}\right)=p_{\mathrm{c}}^{\mathrm{T}} V_{\mathrm{cs}}\left(x_{\mathrm{c}}\right)$, $x_{\mathrm{c}} \in \mathbb{R}^{n_{\mathrm{c}}}$, are positive definite, and noting that $v_{\mathrm{cs}}\left(x_{\mathrm{c}}\right) \leq \max _{i=1, \ldots, q}\left\{p_{\mathrm{c} i}\right\} \mathbf{e}^{\mathrm{T}} V_{\mathrm{cs}}\left(x_{\mathrm{c}}\right)$, where $p_{\mathrm{c} i}$ is the $i$ th element of $p_{\mathrm{c}}$ and $\mathbf{e}=[1, \ldots, 1]^{\mathrm{T}}$, it follows that $\mathbf{e}^{\mathrm{T}} V_{\mathrm{cs}}\left(x_{\mathrm{c}}\right), x_{\mathrm{c}} \in \mathbb{R}^{n_{\mathrm{c}}}$, is positive definite. Now, since $\min _{i=1, \ldots, q}\left\{p_{i} \sigma_{i}\right\} \mathbf{e}^{\mathrm{T}} V_{\mathrm{cs}}\left(x_{\mathrm{c}}\right) \leq p^{\mathrm{T}} \Sigma V_{\mathrm{cs}}\left(x_{\mathrm{c}}\right)$, it follows that $p^{\mathrm{T}} \Sigma V_{\mathrm{cs}}\left(x_{\mathrm{c}}\right)$, $x_{\mathrm{c}} \in \mathbb{R}^{n_{\mathrm{c}}}$, is positive definite. Hence, the function $v\left(x, x_{\mathrm{c}}\right)=p^{\mathrm{T}} V\left(x, x_{\mathrm{c}}\right),\left(x, x_{\mathrm{c}}\right) \in \mathbb{R}^{n} \times \mathbb{R}^{n_{\mathrm{c}}}$, is positive definite. Now, the result is a direct consequence of Theorem 2.7.

(ii) The proof follows from (i) by noting that, for all $i=1, \ldots, q$,

$$
s_{i}\left(u_{i}, y_{i}\right)+\sigma_{i} s_{\mathrm{c} i}\left(u_{\mathrm{c} i}, y_{\mathrm{c} i}\right)=\left[\begin{array}{l}
y \\
y_{\mathrm{c}}
\end{array}\right]^{\mathrm{T}} \tilde{Q}_{i}\left[\begin{array}{l}
y \\
y_{\mathrm{c}}
\end{array}\right] \text {, }
$$

and hence $S(u, y)+\Sigma S_{\mathrm{c}}\left(u_{\mathrm{c}}, y_{\mathrm{c}}\right) \leq \leq 0$. 
For the next result, note that if the discrete-time large-scale nonlinear dynamical sys-

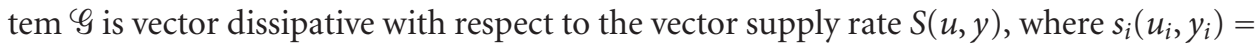
$2 y_{i}^{\mathrm{T}} u_{i}, i=1, \ldots, q$, then with $\kappa_{i}\left(y_{i}\right)=-\kappa_{i} y_{i}$, where $\kappa_{i}>0, i=1, \ldots, q$, it follows that $s_{i}\left(\kappa_{i}\left(y_{i}\right), y_{i}\right)=-\kappa_{i} y_{i}^{\mathrm{T}} y_{i}<0, y_{i} \neq 0, i=1, \ldots, q$. Alternatively, if $\varphi_{\text {is }}$ vector dissipative with respect to the vector supply rate $S(u, y)$, where $s_{i}\left(u_{i}, y_{i}\right)=\gamma_{i}^{2} u_{i}^{\mathrm{T}} u_{i}-y_{i}^{\mathrm{T}} y_{i}$, where $\gamma_{i}>0, i=$ $1, \ldots, q$, then with $\kappa_{i}\left(y_{i}\right)=0$, it follows that $s_{i}\left(\kappa_{i}\left(y_{i}\right), y_{i}\right)=-y_{i}^{\mathrm{T}} y_{i}<0, y_{i} \neq 0, i=1, \ldots, q$. Hence, if $\mathscr{G}_{\mathrm{G}}$ is zero-state observable and the dissipation matrix $W$ is such that there exist $\alpha \geq 1$ and $p \in \mathbb{R}_{+}^{q}$ such that (2.2) holds, then it follows from Theorem 3.8 that (scalar) storage functions of the form $v_{s}(x)=p^{\mathrm{T}} V_{s}(x), x \in \mathbb{R}^{n}$, where $V_{s}(\cdot)$ is a vector storage function for $\mathscr{G}$, are positive definite. If $\mathscr{G}$ is geometrically vector dissipative, then $p$ is positive.

Corollary 6.2. Consider the discrete-time large-scale nonlinear dynamical systems $\mathscr{G}$ and $\mathscr{G}_{\mathrm{c}}$ given by (3.1), (3.2) and (6.1), respectively. Assume that $G_{\mathrm{S}}$ and $\mathscr{G}_{\mathrm{c}}$ are zero-state observable and the dissipation matrices $W \in \mathbb{R}^{q \times q}$ and $W_{\mathrm{c}} \in \mathbb{R}^{q \times q}$ are such that there exist, respectively, $\alpha \geq 1, p \in \mathbb{R}_{+}^{q}, \alpha_{c} \geq 1$, and $p_{\mathrm{c}} \in \mathbb{R}_{+}^{q}$ such that (2.2) is satisfied. Then the following statements hold.

(i) If $\varphi_{G}$ and $\varphi_{\mathrm{c}}$ are vector passive and $\tilde{W} \in \mathbb{R}^{q \times q}$ is asymptotically stable, where $\tilde{W}_{(i, j)} \triangleq$ $\max \left\{W_{(i, j)}, W_{\mathrm{c}(i, j)}\right\}, i, j=1, \ldots, q$, then the negative feedback interconnection of $\varphi_{S}$ and $\mathscr{G}_{\mathrm{c}}$ is asymptotically stable.

(ii) If $\varphi_{\text {and }} \varphi_{\mathrm{c}}$ are vector nonexpansive and $\tilde{W} \in \mathbb{R}^{q \times q}$ is asymptotically stable, where $\tilde{W}_{(i, j)} \triangleq \max \left\{W_{(i, j)}, W_{\mathrm{c}(i, j)}\right\}, i, j=1, \ldots, q$, then the negative feedback interconnection of $\mathscr{G}$ and $\mathscr{G}_{\mathrm{c}}$ is asymptotically stable.

Proof. The proof is a direct consequence of Theorem 6.1. Specifically, (i) follows from Theorem 6.1 with $R_{i}=0, S_{i}=I_{m_{i}}, Q_{i}=0, R_{\mathrm{c} i}=0, S_{\mathrm{c} i}=I_{m_{i}}, Q_{\mathrm{c} i}=0, i=1, \ldots, q$, and $\Sigma=$ $I_{q}$; while (ii) follows from Theorem 6.1 with $R_{i}=\gamma_{i}^{2} I_{m_{i}}, S_{i}=0, Q_{i}=-I_{l_{i}}, R_{\mathrm{c} i}=\gamma_{\mathrm{c} i}^{2} I_{l_{i}}, S_{\mathrm{c} i}=$ $0, Q_{\mathrm{c} i}=-I_{m_{i}}, i=1, \ldots, q$, and $\Sigma=I_{q}$.

\section{Conclusion}

In this paper, we have extended the notion of dissipativity theory to vector dissipativity theory. Specifically, using vector storage functions and vector supply rates, dissipativity properties of aggregate large-scale discrete-time dynamical systems are shown to be determined from the dissipativity properties of the individual subsystems and the nature of their interconnections. In particular, extended Kalman-Yakubovich-Popov conditions, in terms of the local subsystem dynamics and the subsystem interconnection constraints, characterizing vector dissipativeness via vector storage functions are derived. In addition, general stability criteria were given for feedback interconnections of discrete-time largescale nonlinear dynamical systems in terms of vector storage functions serving as vector Lyapunov functions.

\section{Acknowledgment}

This research was supported in part by the Air Force Office of Scientific Research (AFOSR) under Grant F49620-03-1-0178 and the National Science Foundation (NSF) under Grant ECS-0133038. 


\section{References}

[1] M. Araki, Input-output stability of composite feedback systems, IEEE Trans. Automatic Control 21 (1976), no. 2, 254-259.

[2] R. Bellman, Vector Lyapunov functions, J. SIAM Control Ser. A 1 (1962), 32-34.

[3] A. Berman and R. J. Plemmons, Nonnegative Matrices in the Mathematical Sciences, Academic Press, New York, 1979.

[4] D. S. Bernstein and D. C. Hyland, Compartmental modeling and second-moment analysis of state space systems, SIAM J. Matrix Anal. Appl. 14 (1993), no. 3, 880-901.

[5] S. Boyd, L. El Ghaoui, E. Feron, and V. Balakrishnan, Linear Matrix Inequalities in System and Control Theory, SIAM Studies in Applied Mathematics, vol. 15, SIAM, Pennsylvania, 1994.

[6] V. Chellaboina and W. M. Haddad, Stability margins of discrete-time nonlinear-nonquadratic optimal regulators, Internat. J. Systems Sci. 33 (2002), no. 7, 577-584.

[7] L. T. Grujić, A. A. Martynyuk, and M. Ribbons-Parella, Large Scale Systems Stability under Structural and Singular Perturbations, Springer-Verlag, Berlin, 1987.

[8] W. M. Haddad and V. Chellaboina, Nonlinear control of Hammerstein systems with passive nonlinear dynamics, IEEE Trans. Automat. Control 46 (2001), no. 10, 1630-1634.

[9] W. M. Haddad, V. Chellaboina, and E. August, Stability and dissipativity theory for discretetime nonnegative and compartmental dynamical systems, Proceedings of the 40th IEEE Conference on Decision and Control (Orlando, Fla), IEEE Control Systems Society, 2002, pp. 4236-4241.

[10] D. J. Hill and P. J. Moylan, Stability results of nonlinear feedback systems, Automatica 13 (1977), no. $4,377-382$.

[11] V. Lakshmikantham, V. M. Matrosov, and S. Sivasundaram, Vector Lyapunov Functions and Stability Analysis of Nonlinear Systems, Mathematics and Its Applications, vol. 63, Kluwer Academic Publishers, Dordrecht, 1991.

[12] E. L. Lasley and A. N. Michel, Input-output stability of interconnected systems, IEEE Trans. Automatic Control 21 (1976), no. 1, 84-89.

[13] $\longrightarrow L_{\infty}-$ and $l_{\infty}$-stability of interconnected systems, IEEE Trans. Circuits and Systems 23 (1976), no. 5, 261-270.

[14] J. Lunze, Stability analysis of large-scale systems composed of strongly coupled similar subsystems, Automatica 25 (1989), no. 4, 561-570.

[15] A. A. Martynyuk, Stability by Liapunov's Matrix Function Method with Applications, Monographs and Textbooks in Pure and Applied Mathematics, vol. 214, Marcel Dekker, New York, 1998.

[16] _ Qualitative Methods in Nonlinear Novel Approaches to Liapunov's Matrix Functions Dynamics, Monographs and Textbooks in Pure and Applied Mathematics, vol. 246, Marcel Dekker, New York, 2002.

[17] V. M. Matrosov, Method of Lyapunov-vector functions in feedback systems, Automat. Remote Control 33 (1972), no. 9, part 1, 1458-1468 (Russian).

[18] A. N. Michel and R. K. Miller, Qualitative Analysis of Large Scale Dynamical Systems, Mathematics in Science and Engineering, vol. 134, Academic Press, New York, 1977.

[19] D. D. Šiljak, Large-Scale Dynamic Systems. Stability and Structure, North-Holland Series in System Science and Engineering, vol. 3, North-Holland Publishing, New York, 1979.

[20] Complex dynamical systems: Dimensionality, structure and uncertainty, Large Scale Systems 4 (1983), 279-294.

[21] M. Vidyasagar, Input-Output Analysis of Large-Scale Interconnected Systems, Lecture Notes in Control and Information Sciences, vol. 29, Springer-Verlag, Berlin, 1981.

[22] J. C. Willems, Dissipative dynamical systems. I. General theory, Arch. Rational Mech. Anal. 45 (1972), 321-351. 
66 Vector dissipativity and discrete-time large-scale systems

[23] _ Dissipative dynamical systems. II. Linear systems with quadratic supply rates, Arch. Rational Mech. Anal. 45 (1972), 352-393.

Wassim M. Haddad: School of Aerospace Engineering, Georgia Institute of Technology, Atlanta, GA 30332-0150, USA

E-mail address:wm.haddad@aerospace.gatech.edu

Qing Hui: School of Aerospace Engineering, Georgia Institute of Technology, Atlanta, GA 303320150, USA

E-mail address: qing_hui@ae.gatech.edu

VijaySekhar Chellaboina: Mechanical and Aerospace Engineering, University of Missouri-Columbia, Columbia, MO 65211, USA

E-mail address: chellaboinav@missouri.edu

Sergey Nersesov: School of Aerospace Engineering, Georgia Institute of Technology, Atlanta, GA 30332-0150, USA

E-mail address: sergei_nersesov@ae.gatech.edu 